\title{
Study of a pre-Linnaean herbarium attributed to Francesco Cupani (1657-1710)
}

\author{
Santa Pulvirenti, Maria Martina Indriolo, Pietro Pavone \& Rosanna Maria Stefania Costa
}

\begin{abstract}
PULVIRENTI S., M. M. INDRIOLO, P. PAVONE \& R. M. S. COSTA (2015). Study of a pre-Linnaean Herbarium attributed to Francesco Cupani (1657-1710). Candollea 70: 67-99. In English, English abstract. DOI: http://dx.doi.org/10.15553/c2015v701a8

The aim of this work is the study of one of the two pre-Linnaean herbaria belonging to the University of Catania, attributed to Francesco Cupani, a 17th century Sicilian botanist, specifically the volume with the inventory number "VII f2 Hortus Botanicus Catinensis". The two collections were previously unknown and found in 1992 as a result of reorganising the Herbarium of Catania University. The volume examined comprises 164 pages with 1-12 specimens on each sheet. Most likely, some samples come from the Misilmeri Garden of the Prince of Cattolica, to which Cupani dedicated great commitment in its creation, while others may have been acquired from exchanges with contemporary scientists; this is testified by the presence of non-Sicilian or extra-European species and by the extensive correspondence between the Sicilian friar and many contemporary botanists. The material in the herbarium comprises phanerogams (610 angiosperms and 5 gymnosperms), along with a small number of cryptogams (14 pteridophytes, 9 algae and 1 lichen), and 4 marine animals are also present. Unfortunately, some samples are partially or entirely damaged or even missing; nonetheless it has been possible to identify most of them. The Cupani's herbarium is compared with some pre-Linnaean herbaria in order to highlight their main similarities and differences.
\end{abstract}

\section{Keywords}

Cupani - Exsiccata - Herbarium - Pre-Linnaean - Taxonomy

Addresses of the authors:

SP: Department of Biological, Geological and Environmental Sciences, University of Catania, via A. Longo, 19-95125 Catania, Italy. E-mail: sgpulvirenti@gmail.com MMI, PP, RMSC: Department of Biological, Geological and Environmental Sciences, University of Catania, via A. Longo, 19-95125 Catania, Italy.

Submitted April 22,2014. Accepted on April 16, 2015.

Edited by F. Stauffer \& P. Bungener 


\section{Introduction}

The 17th century Sicilian botanist Francesco Cupani was born in 1657 in Mirto, a small village in the Messina province. He studied theology and then joined the Franciscan order. After a period of teaching in Verona he returned to Sicily and dedicated himself to the study of botany. His interest in plants was influenced by the friendship with Paolo Boccone, naturalist and botanist of good fame in Europe (Mongitore, 1707; Russo, 1819). Cupani was the author of important floristic works (Cupani, 1692, 1695, 1696, 1697) and of the unfinished "Panphyton siculum". Thanks to the patronage of the first Prince of Cattolica, he contributed with great commitment to the creation of a Botanic Garden at Misilmeri (hamlet eight miles from Palermo), known as "Hortus Catholicus" and destined to collect plants for medicinal uses of public interest. With the support of some druggists, as Pietro Citraro and Francesco Scaglione, and with the financial help of the Prince of Cattolica, Cupani contributed to enrich the Garden of Misilmeri with many species coming not only from Sicily but also from others territories (CUPANI, 1696).

Cupani lived in a small Sicilian village (Misilmeri) and he did not belong to any academic circle. Nevertheless, mostly thanks to the Prince of Cattolica, he kept contacts with the most important scientists of his time: William Sherard (English botanist, 1659-1728), John Ray (English naturalist, 1627-1705), Caspar Commelin (Dutch botanist, 1668-1731), Joseph Pitton de Tournefort (French botanist, 1656-1708), Giovanni Battista Trionfetti (Italian botanist, 1656-1708), Johann Georg Volkamer (German naturalist, 1616-1693) and Johannes Böhm (German physician, 1640- ca. 1731). Remarkably thanks to the extensive correspondence with Sherard, he participated in the debate on the method of naming plants (Arber, 1912). Cupani and his contemporaries did not succeed in devising a "taxonomic system", although some of them have made significant attempts (GREUTER et al., 2005). The pre-Linnaean botanists certainly contributed to lay the foundations of "modern scientific knowledge", as Linnaeus himself acknowledged in classifying them as among the "Curious" (Linnaeus, I 736). Linnaeus used the category of the "Curious" to label a group of authors as important precursors of modern botany. The category has recently been reused by DAston (2012) to define the non-academic culture of the 1600's that played an important role in circulating the "new method" (Pulvirenti et al., 2015, in press). Cupani died in Palermo in 1710.

\section{Cupani and the exchange of information}

Thanks to the exchange of illustrated publications, seeds and in particular dried specimens, Cupani developed a real "scientific network" that enabled botanical material to circulate for examination and comparison. The novelty of these scientific exchanges practised by him and other contemporary botanists can be summarised precisely in the extraordinary ability to build a "scientific network" by making use of all the tools the times allowed (Pulvirenti et al., 2015, in press).

The conditions favouring the circulation of information were the development of maritime communications in the Mediterranean, the decrease of risk of piracy, the presence of a widespread consular network and the possibility of travelling through Europe and beyond. Alongside exchanging boxes of seeds and publications (replete with drawings and descriptions), the exchange of Horti Sicci represented one of the main tools for developing discussions among the scientists of the period and standardizing classifications. Printing a volume of iconography, was a weighty commitment and the dramatic history of the "Panphyton siculum" (never published, though nearly all the images were engraved) points out this aspect. It should be highlighted that even Linnaeus, praising those he called the "Curious", makes explicit reference to the merit of many scientists who popularized, at their own expense, the knowledge and comparison of plants (LinnaEus, 1736). It was much simpler (and also more useful) to circulate collections of exsiccata, which were easier to compare, study and classify, than iconographic documents.

\section{The use of herbaria in scientific communication}

The herbaria gathered by Cupani were made with samples collected from the Misilmeri Garden or surrounding areas; it was apparently a thorough task and certainly could not be carried out by only one person. From Cupani's letters, an intensive system of exchanges (in particular) of herbaria with the main European scientists emerges with clarity; moreover, there were collaborators working under his supervision, as might be interpreted from the letter sent by Sherard, dated 18 October 1696, in which he asked the Sicilian friar to prepare an herbarium (Dollo, 1979).

The production of herbaria continued long beyond Cupani, as is demonstrated in the correspondence between the Prince of Cattolica (the patron of the Misilmeri Garden) and the scientists of the court of Cosimo III de' Medici, twenty years or so after the death of the Franciscan friar (TARgioni-Tozzetti, 1858; Baroni, 1896; Mattei, 1906). However, the observations of MATTEI (1906) may be a useful indication for questioning the direct attribution of all the "Herbaria of the Prince of Cattolica" to Cupani. It is perhaps more realistic to imagine a kind of "laboratory" settled and directed, at least during an early stage, by Cupani and afterwards continued and managed by his "collaborators". It cannot be ruled out that herbaria that might be attributed to what must have been the "laboratory" of the Misilmeri Garden are present in the collections of the most important museums. From time to time, often accidentally, as in our case, some previously unknown collections were discovered (Brullo \& Pavone, 1993; Mazzola \& Raimondo, 1995). Cupani indicated brief descriptions of the plants that he had studied. However, those descriptions 


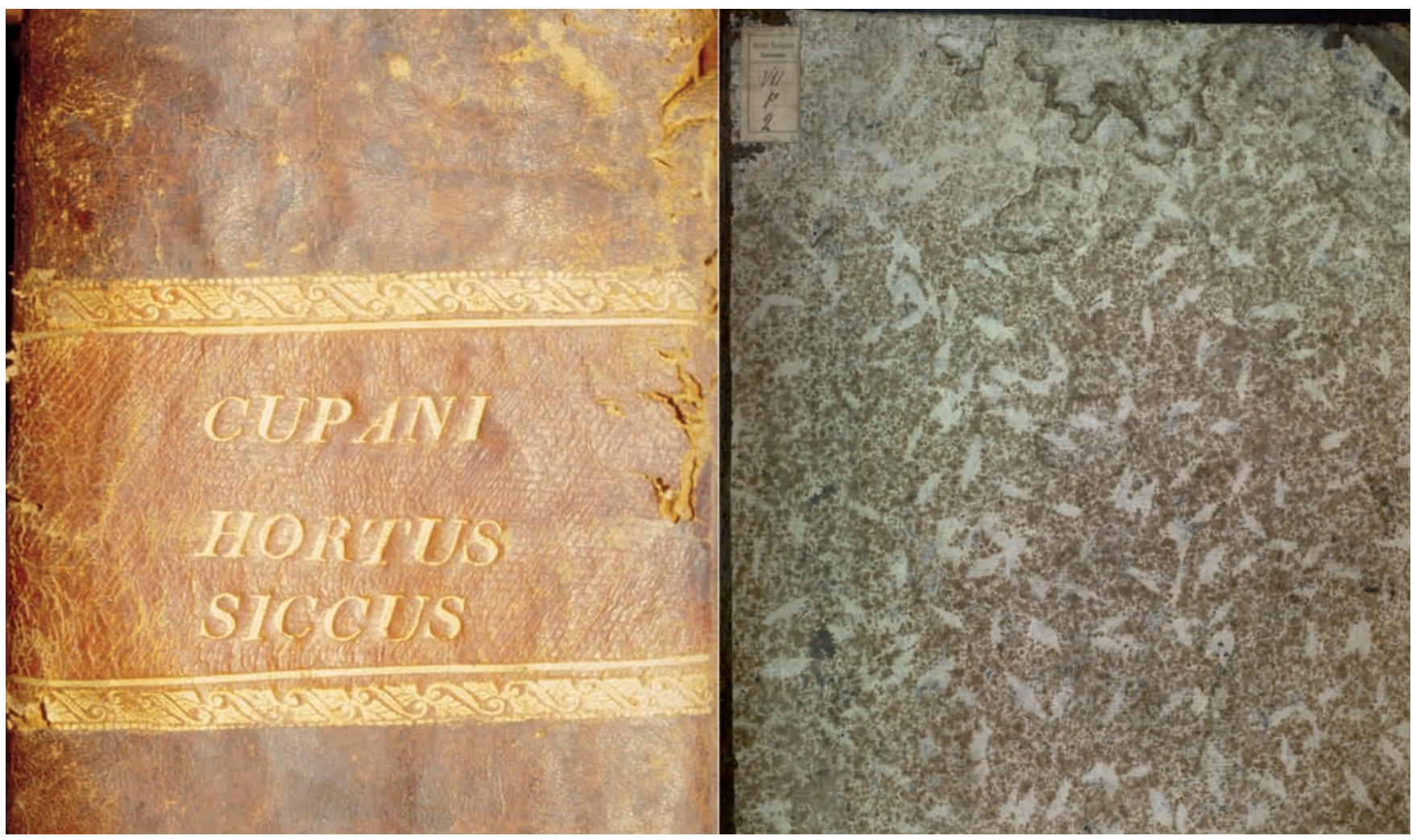

Fig. 1.-Spine and inventory label of the Cupani Herbarium.

cannot be equated with Linnaean binomials (genus, species). Cupani called his descriptions "polilogus", i.e. sentences of a few words (Cupani, 1692, 1695, 1696, 1697). His technique matched a simple requirement of synthesis. Instead, the Linnaean binomial represents a classification system allowing the identification of a genus and a species in a frame of reference (Pulvirenti et al., 2015, in press).

\section{Cupani's herbaria of Catania University}

On the basis of these considerations it is therefore possible to frame the function of the two herbaria belonging to the Department of Biological, Geological and Environmental Sciences, found by chance in 1992 (Brullo \& Pavone, 1993). These collections are presented as two volumes of exsiccata attributed to Francesco Cupani, of different size and structure, without any information on when they were made. The hypothesis of attributing these herbaria to Cupani derives both from the explicit reference in the title-page as well as the reconstruction of the several transfers of ownership. In the literature, the citations regarding these "Horti Sicci" are rather scant. Scıñ̀ (1824), albeit speaking more generally of Cupani, simply states that Giovanbattista Caruso "took the trouble to collect all Cupani's papers and kept only the herbarium for himself; for this reason it is nowadays stored in Catania along with Caruso's books" (ScıNÀ, 1824; citation translated).
More information was supplied by Salvatore Portal who was a physician-botanist and the holder of a well-known botanical garden on the outskirts of Biancavilla. In his paper of 1836 he mentioned two volumes of exsiccata made by Cupani and claimed that he inherited one of them from his father, who had in turn acquired it in Palermo from the chemist Don Giuseppe Chiarelli (Portal, 1836). This volume was entire and in good condition, with the exception of the "title-page and some blank pages at the end". Portal also pointed out that the other volume, with the title-page "Hortus Catholicus species plantarum autografo Fran. Cupani", may be found in the library of the Catania University and was not complete as it only contained 190 pages. Portal compared the two volumes and noted that the plants found in his herbarium and those of the Catania University were different in terms of the number of species and arrangement; moreover, the two volumes differed in format and kind of paper used. He also stated that in his volume the plants came from the Garden of the Prince of Cattolica as well as from other localities, whereas the volume stored at the University contained plants exclusively from the Misilmeri Garden. The two volumes found in 1992 and currently kept in the herbarium of the Department of Biological, Geologic and Environmental Sciences (CAT), match well Portal's description. The first volume appears to have been entrusted 


\section{PLANTA SICCA}

QUA OMNES IN HORTO CATHOLICO crescunt

ET MULTA ALIAE QUAE STCILIAE niscuntux terris plexisque lacis AUGTORE FRANCISCO CUPANI SAC.THEOL. MAGTSTRO SICULO A MYRTHENSI

\section{PANORMO}

Fig. 2. - Title page of the Cupani Herbarium. 
in 1912 to the Director of the Botanical Institute, Luigi Buscalioni (1863-1954), by the Royal University Library of Catania. The second volume, subject of the present study, is larger than the former and is in a fair state of preservation; it comprises 164 pages, largely containing exsiccata of phanerogams along with some specimens of algae, lichens, pteridophytes and a few marine animals. On the first page of this volume there is a handwritten note by Prof. Emilio Chiovenda (1871-1941), the Director of the Institute of Botany from 1926 to 1929 , which helps us in tracing its history. It reads: "This herbarium of C. F. Cupani comes from the library of Doctor Salvatore Portal, minister of Biancavilla; I purchased it on 9.3.1927 from the Tirelli bookshop in Catania" (translated). It is unknown how the herbarium passed from the hands of Salvatore Portal to the Tirelli bookseller, which sold it afterwards to Prof. Chiovenda.

The aim of this work is to study one of the two aforementioned pre-Linnaean herbaria attributed to F. Cupani, specifically the volume with the inventory number "VII f2 Hortus Botanicus Catinensis" (Fig. 1-3). This volume was digitized in 2005 under the supervision of Prof. Pietro Pavone in the frame of the initiative "Coordinated Project CataniaLecce 2000-2006, step 8, Botanic Garden and Herbarium”; this initiative was intended to enhance the cultural heritage of Sicily. Pictures of all samples include in this herbarium are available online at http://www.dipbot.unict.it/erbario/cupani/ index.html.

\section{Material and methods}

A list of all specimens associated to Cupani's historical herbarium was prepared (Table 1), including for each sample the following information:

a) number of the herbarium sheet under which the sample is currently placed;

b) Literal transcription of original name proposed in the herbarium (polilogus/polynomial) and local dialect when present. The absence of the name is indicated by " "," any illegible writing by "?" and the fact that the name noted was transcribed from the index at the end of the volume of exsiccata by "index";

c) Current taxonomic identification. Regarding angiosperms, gymnosperms and pteridophytes we have followed treatments such as EURO+MED (2006) and partially Giardina et al. (2007) and Pignatti (1982). Thus the absence of a scientific name is indicated by "/", when we were unable to identify the taxon;

d) state of preservation of the specimen according to three categories: bad (if there are only plant fragments), poor (if the sample is damaged) and good (if the sample displays all its parts). A missing sample is also indicated in the event that only remaining marks or the strip of paper by which it was attached to the sheet were observed.
A list of alphabetically arranged taxa is presented (Appendix 1); this list includes plants of the studied herbarium. Using on-line data (see below) the Cupani's herbarium is compared with some pre-Linnaean herbaria in order to highlight their main similarities and differences.

\section{Results}

The volume of exsiccata studied comprises 164 sheets (numbered to 163 but number 57 was erroneously assigned to two different sheets), numbered at the top right corner of each sheet. At the end of the volume there is an index listing nearly all the plants contained in this herbarium arranged by first letter alphabetical order (Fig. 4). Next to, or below, every sample there are original Latin handwritten notes and, sometimes vernacular names. Next to each sample, most probably corresponding to a later addition, a red and blue number written in pencil was made by an unknown hand. In addition, from sheet 108 to 118, another unidentified writer added the correct name of the species in pencil. On every sheet there are between 1 to 12 specimens, depending on the dimensions of the sample (Fig. 5, 6). The samples are arranged on one side of every sheet and fixed with irregularly cut strips of paper and glue. We were not able to detect whether this corresponds to the original mounting proposed for the herbarium or whether it was proposed during a later organisation. The doubt remains because in some cases the imprint of the plant can be seen in a different position; besides it is not known if the system of fixing the specimens with strips was original or alternative to the method using the glue. Either way, it is sure that the plant specimens associated to the herbarium sent to Micheli by the Prince of Cattolica in 1733 were simply glued (BARONI, 1896).

Regarding the nomenclature associated to the specimens, some taxa belonging to the same species have different names (i.e. Anthyllis vulneraria L. or Ononis natrix L.), while others are classified under the same name (i.e. Stachys ocymastrum (L.) Briq. or Ranunculus flammula L.). Several reasons suggest that the herbarium was probably re-bound in fairly recent times. In the first place, the binding is entirely different from the one observed in the other volume, the latter believed to still present the original type of binding. Secondly, leafing through the volume one may see that the sheets have been cut at the top and bottom, and perhaps also at the edges; in some cases parts of the samples and/or the numbering are missing. Thirdly, on the back of the volume the sentence "Cupani Hortus Siccus" is written in gold and with characters almost certainly not corresponding to the end of the $17^{\text {th }}$ century.

The page preceding the title-page shows a handwritten note attributed to Chiovenda regarding the transfer of ownership of the volume. The title on this page reads: "plantae siccae - quae omnes in Horto Catholico - crescunt - et multae aliae quae Siciliae - nascuntur terris plerisque locis - auctore Francisco Cupani - sac. theol. magistro siculo - a Myrthensi 


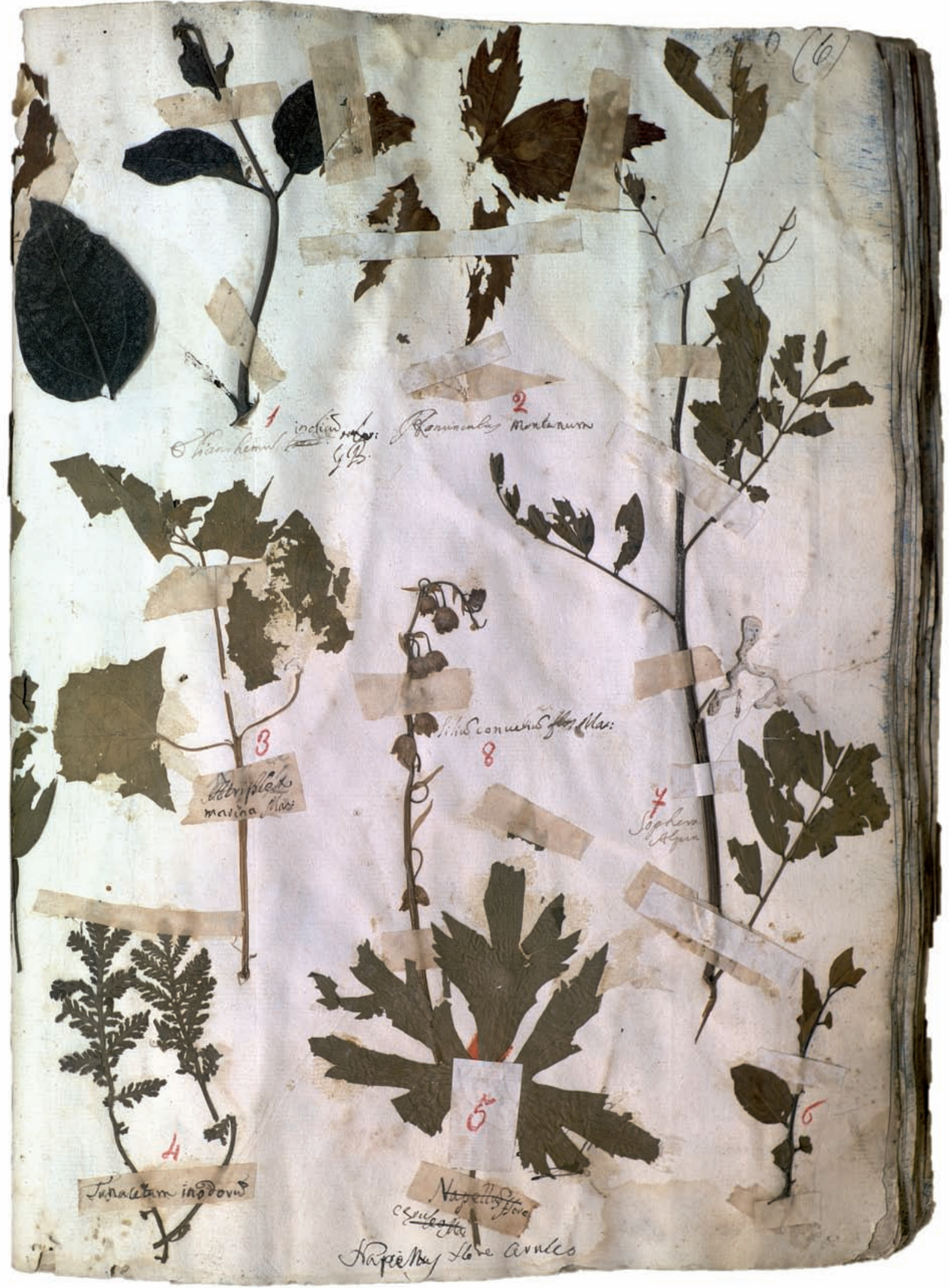

Fig. 3. -Sheet number 6 of the Cupani Herbarium. 


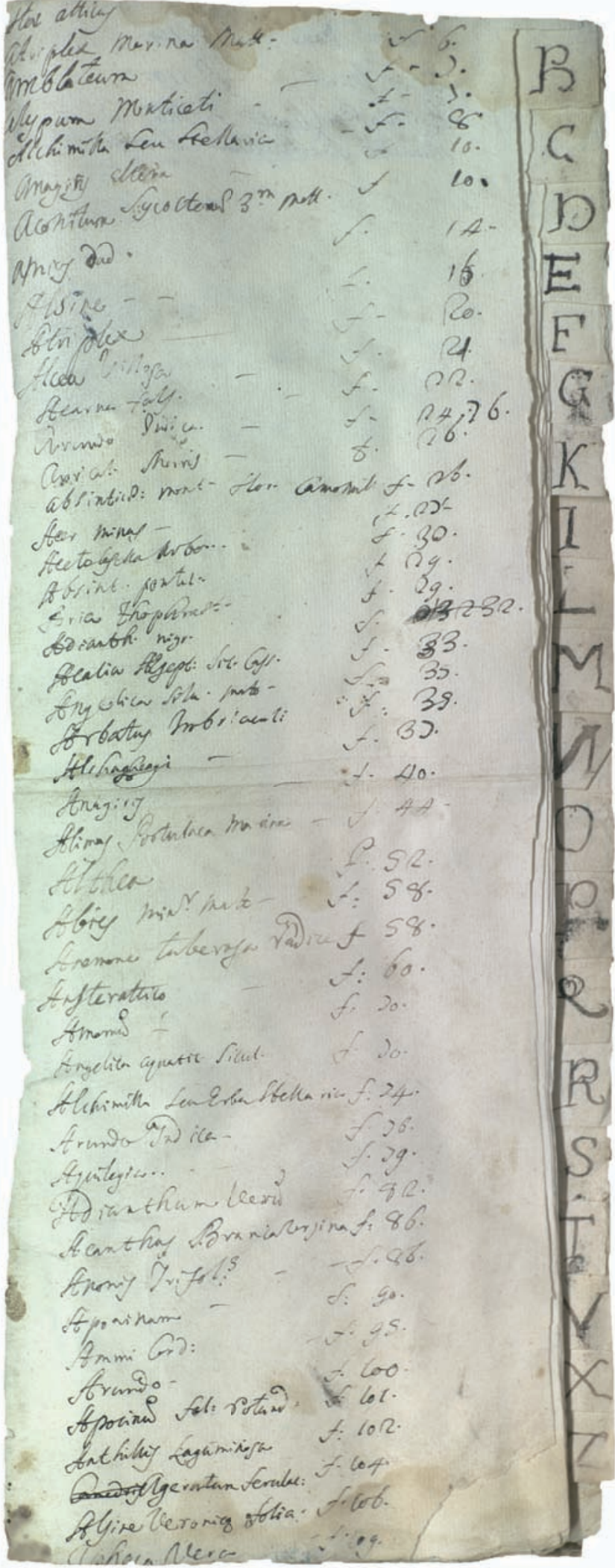

Fig. 4. - First page of the index of the Cupani Herbarium.
- Panormo". However, according to Portal (1836), this note may not be original but added later. Between the title-page and the first sheet, there is a coloured tissue overlay that must have been used to protect the dried plants that are highly fragile. Presumably, this protection is not original either. The herbarium is made up of 669 samples. The specimens are distributed as follow: 610 (91.18\%) are angiosperms, 14 (2.09\%) are pteridophytes, $9(1.35 \%)$ are algae, $5(0.75 \%)$ are gymnosperms, $4(0.6 \%)$ are animals, $1(0.15 \%)$ is a lichen and 26 (3.89\%) are missing. Most samples (407, equal to 60.84\%) are in a good state of conservation, $181(27.06 \%)$ in a poor state and 55 (8.22\%) in bad condition (Fig. 7).

\section{Comparisons with other pre-Linnaean herbaria}

Between the late 1600s and the mid-1700s there were numerous "pre-Linnaean" herbaria in Europe, among which should be highlighted: Sherard's herbarium, Bobart the Youger's herbarium, Sloane's herbarium, Clifford's herbarium, Hermann's herbarium, Helwing's herbaria, Münchenbergs's herbarium, and the Salvador's herbarium (see description below). Most of these herbaria have been studied because they are historically interesting from the viewpoint of nomenclature and floristic biodiversity of the pre-Linnaean period (IBÁÑ̃Z et al., 2008; Menezes de Sequeira et al., 2010; Santos-Guerra et al., 2011; Andel et al., 2012; Spalik, 2014). It is difficult to propose thorough comparisons between the herbarium subject of our study to other pre-Linnaean herbaria, especially since most them have been widely restored and/or increased in later periods. The greatest value of Cupani's herbarium is the fact that it has undergone very few rearrangements. Indeed, with the exception of the binding and notes written in pencil, it appears to us being in its original form. Despite these difficulties, here below we highlight some similarities and differences identified between Cupani's herbarium and other contemporary pre-Linnaean herbaria:

\section{Cupani's herbarium}

Repository: Catania University.

Period of collection: late 1600 .

Rearrangements: few (later binding and notes written in pencil).

Bookbinding: single volume.

Total number of specimens: 669.

Specimens on each sheet: 1-12.

Mounting method: strips of paper and glue.

Nomenclature: Latin polynomial (few words) and sometimes vernacular names.

On-line access: http://www.dipbot.unict.it/erbario/ cupani/index.html. 


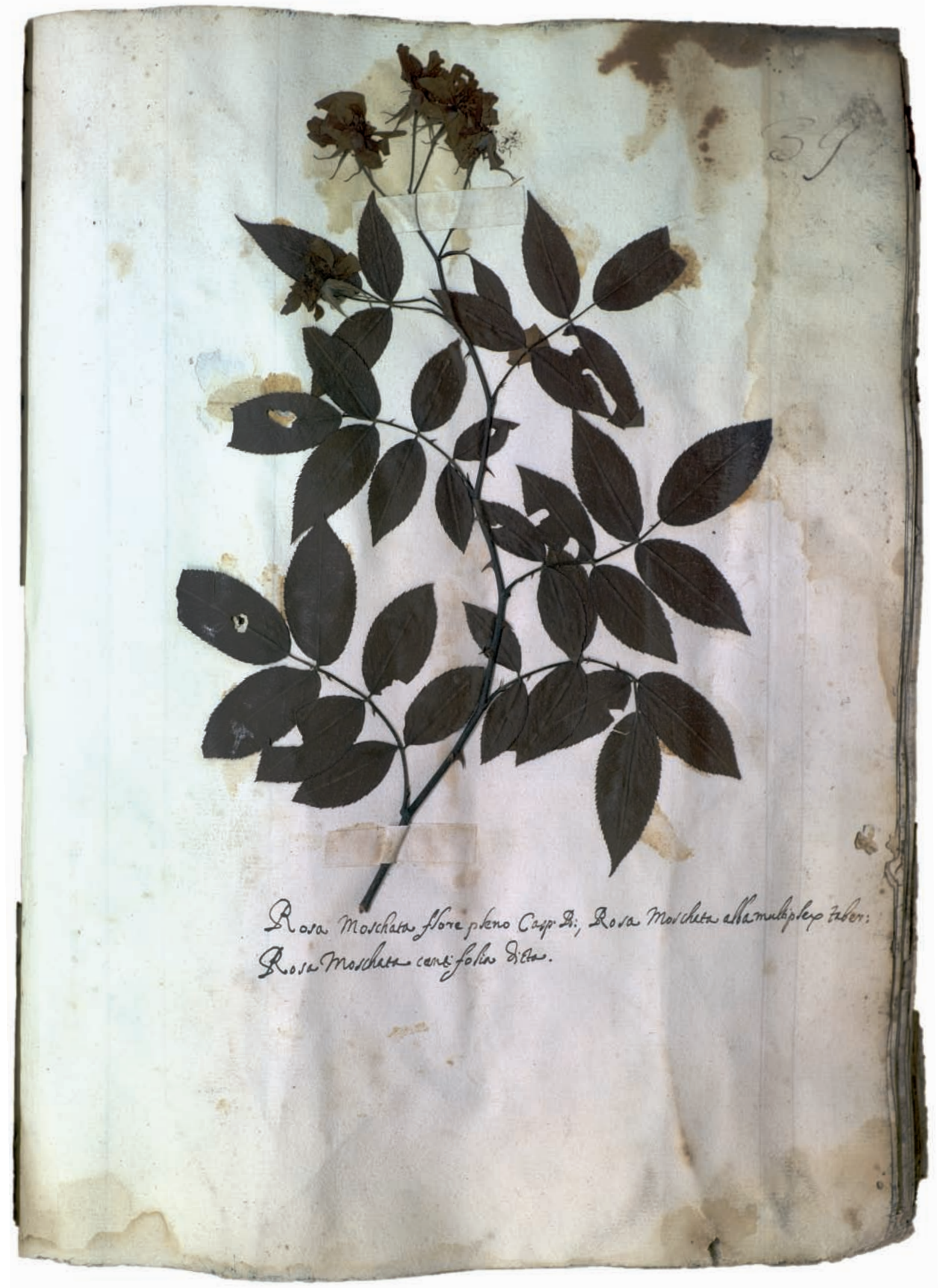

Fig. 5. - Sheet number 39 of the Cupani Herbarium containing one specimen of Rosa L. (Rosaceae). 


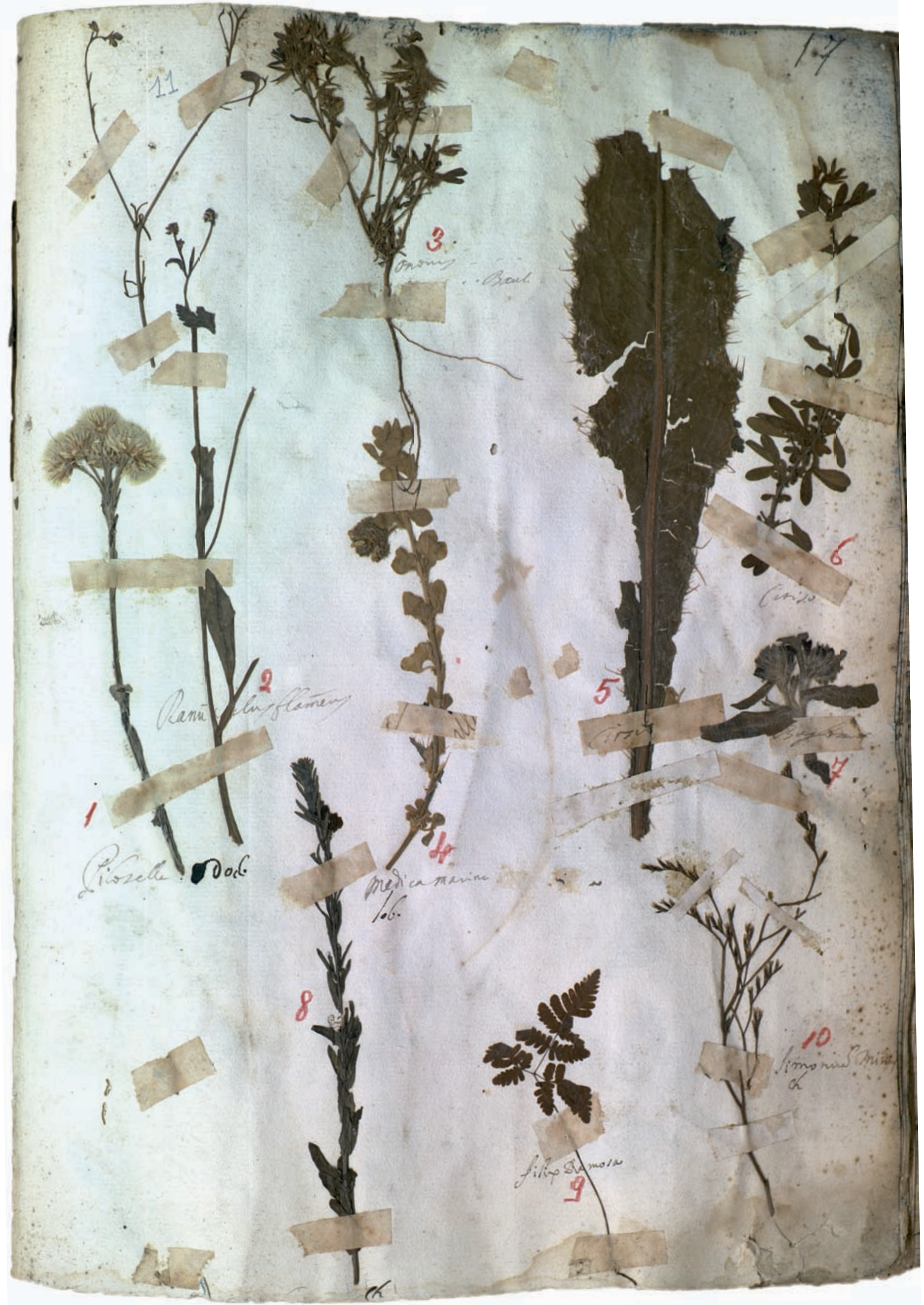

Fig. 6. - Sheet number 17 of the Cupani Herbarium. 


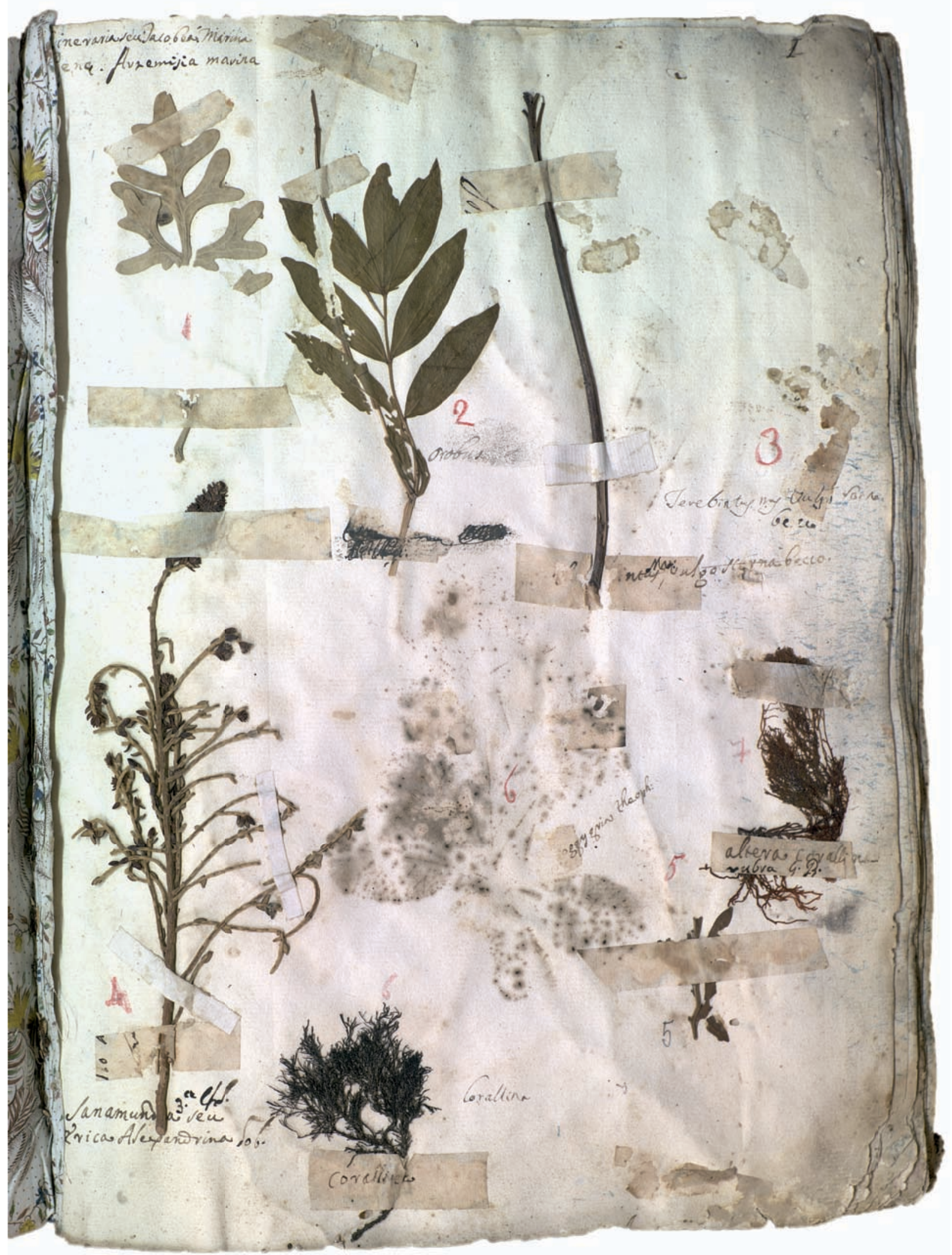

Fig. 7. - Sheet number 1 of the Cupani Herbarium. 


\section{Sherard's herbarium}

Repository: Oxford University Herbaria.

Period of collection: from ca. 1680 .

Rearrangements: augmented until 1796, rearranged by Dillenius from 1721, by G. C. Druce in the late nineteenth century and, by other botanists during the twentieth century.

Bookbinding: individual sheets.

Total number of specimens: about 20,000.

Specimens on each sheet: usually one.

Mounting method: unknown original arrangement.

Nomenclature: Latin polynomial (few words).

On-line access: http://herbaria.plants.ox.ac.uk/bol/

Sherard/Pages/DBnotes.

\section{Bobart the Youger's herbarium}

Repository: Oxford University Herbaria.

Period of collection: from ca. 1666.

Rearrangements: rearranged probably by G. C. Druce.

Bookbinding: originally bounded, but now stored as separate sheets in Solander boxes.

Total number of specimens: 2202.

Specimens on each sheet: usually one.

Mounting method: glued plants.

Nomenclature: Latin polynomial (several words) and sometimes English common names.

On-line access: http://herbaria.plants.ox.ac.uk/bol/ bobart.

\section{Sloane's herbarium}

Repository: Natural History Museum (London).

Period of collection: ca. 1687-1689.

Rearrangements: most likely rearranged.

Bookbinding: seven volumes.

Total number of specimens: 1275 .

Specimens on each sheet: usually one.

Mounting method: strips of paper and glue.

Nomenclature: Latin polynomial (many words).

On-line access: http://www.nhm.ac.uk/ research-curation/scientific-resources/collections/ botanical-collections/sloane-herbarium/.

\section{Clifford's herbarium}

Repository: Natural History Museum (London).

Period of collection: 1720 s.

Rearrangements: most likely original.

Bookbinding: single unconnected sheets.

Total number of specimens: 3447 .

Specimens on each sheet: usually one (many specimens mounted such that they appear to be growing out of an engraved paper urn)

Mounting method: strips of paper and glue.

Nomenclature: Latin polynomial (few words), sometimes inscribed on ornate engraved label.

On-line access: http://www.nhm.ac.uk/researchcuration/scientific-resources/collections/botanicalcollections/clifford-herbarium/index.html.

\section{Hermann's herbarium}

Repository: Natural History Museum (London).

Period of collection: ca. 1672-1677.

Rearrangements: most likely original.

Bookbinding: four volumes of dried plants and one of illustrations.

Total number of specimens: 1215 .

Specimens on each sheet: frequently several.

Mounting method: strips of paper and glue.

Nomenclature: Latin polynomial (many words) and often Sinhalese names.

On-line access: http://www.nhm.ac.uk/researchcuration/scientific-resources/collections/botanicalcollections/hermann-herbarium/index.html.

\section{Helwing's herbarium}

Repository: Polish National Library and University of Warsaw.

Period of collection: ca. 1695-1705.

Rearrangements: most likely original.

Bookbinding: two volumes.

Total number of specimens: about 1400 .

Specimens on each sheet: usually few.

Mounting method: glued plants. 
Nomenclature: Latin polynomial (few words) and sometimes Polish and German common names.

On-line access: http://polona.pl/item/7971108/0/; http://blog.polona.pl/2014/10/zielnik-helwinga-i/.

\section{Münchenbergs's herbarium}

Repository: Swedish Museum of Natural History (Stockholm).

Period of collection: ca. 1699-1702.

Rearrangements: most likely original.

Bookbinding: one small volume.

Total number of specimens: about 300 .

Specimens on each sheet: usually many.

Mounting method: fixed by a double hole.

Nomenclature: Latin polynomial (many words) and Swedish and German common names.

On-line access: http://www.nrm.se/ forskningochsamlingar/botanik/fbokbo/botaniskhistoria/ antoniusmunchenbergsherb.528.html.

\section{Salvador's herbarium}

Repository: University of Barcelona.

Period of collection: ca. 1700-1745.

Rearrangements: rearranged by Pourret in 1782 .

Bookbinding: unknown.

Total number of specimens: 4026 sheets.

Specimens on each sheet: usually few.

Mounting method: sewn using needle and thread.

Nomenclature: Latin polynomial (many words).

On-line access: http://www.ibb.bcn-csic.es/herb_ historics_ang.html\#Salvador.

\section{Conclusions}

In the light of these results, it may be affirmed that specimens associated to the Cupani's herbarium are well preserved, despite the damage over time and the several transfers of ownership. Regarding the taxonomic composition of the herbarium there is an evident prevalence of angiosperms (more than 400 taxa), which are very well-represented according to their species number. In addition to many specimens of Sicilian origin, a certain number are from other geographic regions and some are also exotic (to the Italian flora), probably deriving from exchanged seeds.

The volume of exsiccata studied is of special interest for several reasons. In the first place it is a precious historical document, since it provides a detailed picture of the floristic biodiversity of the period. In the second, regarding the names attributed to the samples, it represents an example of pre-Linnaean attempts to simplify and standardise botanical nomenclature. Moreover the study of this herbarium certainly contributes to our knowledge of pre-Linnaean herbaria as regards the preservation techniques, classification and exchange of plants, which contributed to the origins and development of modern botany.

\section{Acknowledgments}

The authors thank Prof. S. Brullo for the critical review of the manuscript, S. Conway for translating the article and Prof. A. Brayley for correcting the English. An anonymous reviewer kindly provided valuable comments to an advanced version of the manuscript. 


\section{References}

Andel van, T., S. Veldman, P. Maas, G. Thijsse \& M. Eurlings (2012). The forgotten Hermann Herbarium: a 17th century collection of useful plants from Suriname. Taxon 61: 1296-1304.

Arber, A. (1912). Herbals, their origin and evolution, a chapter in the history of botany, 1470-1670. The University Press.

Baroni, E. (1896). Illustrazione di un Orto Secco del Principe della Cattolica, da questi donato a Pier Antonio Micheli nell'anno 1733. Nuovo Giorn. Bot. Ital. 3: 439-468.

Brullo, S. \& P. Pavone (1993). On Francesco Cupani's Hortus Siccus and its scientific significance. Webbia 48: 539.

CupAni, F. (1692). Catalogus plantarum Sicularum noviter adinventarum. Palermo.

Cupani, F. (1695). Sillabus Plantarum Siciliae, nuper detectarum. Palermo.

Cupani, F. (1696). Hortus Catholicus, cum supplemento ad eundem Hortum. Napoli.

Cupani, F. (1697). Supplementum alterum ad Hortum Catholicum. Palermo.

Daston, L. (2012). Curiosità e studio della natura. In: Petruccioli, S. et al. (ed.), La rivoluzione scientifica. Luoghi e forme della conoscenza: 3654-4138. (Collana di Storia della Scienza), Treccani, [Kindle Edition].

Dollo, C. (1979). Filosofia e scienze in Sicilia. Cedam, Padova.

Euro+Med (2014). Euro+Med PlantBase - the information resource for Euro-Mediterranean plant diversity [http://ww2.bgbm.org/ EuroPlusMed/].

Giardina, G., F. M. Raimondo \& V. Spadaro (2007). A catalogue of plants growing in Sicily. Bocconea 20.

Greuter, W., M. Aghababian \& G. Wagenitz (2005). Vaillant on Compositae - systematic concepts and nomenclatural impact. Taxon 54: 149-174.

Ibáñez, N., J. M. Montserrat \& I. Soriano (2008). Type specimens of names of species authored by Pourret conserved in the Salvador herbarium (BC). Taxon 57: 633-636.
Linnaeus, C. (1736). Biblioteca Botanica. Amsterdam.

Mattei, G. E. (1906). Un altro Erbario dell'Orto Cattolico. Boll. Regio Orto Bot. Palermo 5: 22-45.

Mazzola, P. \& F. M. Raimondo (1995). The Cupani's "Hortus siccus Principis Catholicae" in the Istituto Agrario Castelnuovo Library. Palermo. Giorn. Bot. Ital. 129: 159.

Menezes de Sequeira, M., A. Santos-Guerra, C. E. Jarvis, A. Oberli, M. A. Carine, M. Maunder \& J. Francisco-Ortega (2010). The Madeiran plants collected by Sir Hans Sloane in 1687, and his descriptions. Taxon 59: 598-612.

Mongitore, A. (1707). Bibliotheca sicula sive de scriptoribus siculis. Vol. I. Palermo.

Pignatti, S. (1982). Flora d'Italia. Ed Agricole, Bologna.

PorTAL, S. (1836). Cenni sopra la virtù medica delle mandorle, della celidonia maggiore e del crescione acquatico. Palermo.

Pulvirenti, S., R. M. S. Costa \& P. Pavone (2015, in press). Francesco Cupani: the scientific "network" of his time and the making of the Linnaean "system". Acta Bot. Gallica: 162(2).

Russo, G. (1819). Biografia degli uomini illustri della Sicilia. Vol. III. Napoli.

Santos-Guerra, A., C. E. Jarvis, M. A. Carine, M. Maunder $\&$ J. Francisco-Ortega (2011). Late17th century herbarium collections from Canary Islands: the plants collected by James Cuninghame in La Palma. Taxon 60: 1734-1753.

ScInÀ, D. (1824). Prospetto della storia letteraria di Sicilia nel secolo decimottavo. Vol. I. Palermo.

Spalik, K. (2014): Pre-Linnaean herbaria viva of Helwing in the collections of the National Library of Poland and the University of Warsaw. Acta Soc. Bot. Poloniae 83: 13-16.

Targioni-Tozzetti, G. (1858). Notizie della vita e delle opere di Pier Antonio Micheli. Le Monnier. Firenze. 
Table 1. - List of the samples contained in the Cupani Herbarium.

\begin{tabular}{|c|c|c|c|}
\hline $\begin{array}{l}\text { Herbarium } \\
\text { sheet number }\end{array}$ & $\begin{array}{l}\text { Plant name as indicated } \\
\text { in the Herbarium }\end{array}$ & Current taxonomic identification & $\begin{array}{c}\text { State of } \\
\text { preservation }\end{array}$ \\
\hline 1 & / & / & bad \\
\hline 1 & Altera corallina rubra G. B. & $/$ & good \\
\hline 1 & Corallina & / & good \\
\hline 1 & Coggygria Teoph. & / & no sample \\
\hline 1 & Orobus & Lathyrus sp. & poor \\
\hline 1 & Terebintus mas vulgo scornabecco & Pistacia terebinthus L. & bad \\
\hline 1 & Cineraria seu Jacobea marina ?Artemisia marina & Senecio sp. & bad \\
\hline 1 & Sanamunda seu Erica Alessandrina Lob. & Thymelaea passerina (L.) Coss. \& Germ. & good \\
\hline 2 & Alectorolofos seu fistularia & / & poor \\
\hline 2 & Tragopogon purpureum Col. & / & bad \\
\hline 2 & Tragopogon Col. & / & bad \\
\hline 2 & Moluccha aspera & / & no sample \\
\hline 2 & Olea boemica & / & bad \\
\hline 2 & $?$ & / & no sample \\
\hline 2 & Pseudodictamnus Dod. & Ballota pseudodictamnus (L.) Benth. & good \\
\hline 2 & Campanula arvensis Dod. sive speculus veneris & Falcata legousia (Ten.) Janch. & good \\
\hline 2 & Medica marina Lob. & Medicago marina $\mathrm{L}$. & good \\
\hline 2 & Tragoriganus & Micromeria sp. & poor \\
\hline 3 & Marina? & / & no sample \\
\hline 3 & $?$ & / & no sample \\
\hline 3 & Barba jovis C.D. & Anthyllis barba-jovis L. & good \\
\hline 3 & Sive Laudanum Matt. ? & Cistus monspeliensis L. & good \\
\hline 3 & Alcea villosa Dalicampij Chab. Lug. & Malva cretica Cav. & poor \\
\hline 3 & Helicrisus Syl: C.B. & Phagnalon sordidum (L.) Rchb. & good \\
\hline 3 & Cardus erectus seu Acarna valerandi & Picnomon acarna (L.) Cass. & good \\
\hline 4 & lacea centauroides & / & no sample \\
\hline 4 & / & / & no sample \\
\hline 4 & Cariophillata Matt. & / & poor \\
\hline 4 & Gramen Alopiuros & Cynosurus echinatus $\mathrm{L}$. & good \\
\hline 4 & Critmum quartus Matth. & Falcaria vulgaris Bernh. & poor \\
\hline 4 & Oculus Xrhisti seu Aster? & Inula oculus-christi L. & good \\
\hline 4 & Natrix Plinii seu Ononis no spinosa lutea & Ononis natrix L. subsp. natrix & good \\
\hline 4 & Senecio & Senecio sp. & good \\
\hline 4 & Senecio minor vulgaris C.B. & Senecio vulgaris $\mathrm{L}$. & poor \\
\hline 5 & Aria sive Sorbus? & / & no sample \\
\hline 5 & Altera corallina rubra & / & no sample \\
\hline 5 & Ranunculus Narcisi flore Clus & Aconitum sp. & good \\
\hline 5 & Pseudodictamus verticillatus inodorus ?dictamus Dod. & Ballota pseudodictamnus (L.) Benth. & good \\
\hline 5 & Gramen cyperoides & Carex cf. vulpina L. & good \\
\hline 5 & Clinopodius pm Matt. & Clinopodium vulgare L. & good \\
\hline 5 & Limonius parvulus? & Limonium sp. & good \\
\hline 5 & ?Marinus ? Caspari Bahuini & Achillea maritima (L.) Ehrend. \& Y. P. Guo & good \\
\hline
\end{tabular}




\begin{tabular}{|c|c|c|c|}
\hline $\begin{array}{l}\text { Herbarium } \\
\text { sheet number }\end{array}$ & $\begin{array}{l}\text { Plant name as indicated } \\
\text { in the Herbarium }\end{array}$ & Current taxonomic identification & $\begin{array}{c}\text { State of } \\
\text { preservation }\end{array}$ \\
\hline 5 & Peucedanum & Peucedanum sp. & bad \\
\hline 5 & Elichriso ? C.B. & Phagnolon saxatile (L.) Cass. & good \\
\hline 5 & Polygala ? Ch. sive Polygala ? Lob. & Polygala preslii Spreng. & poor \\
\hline 6 & Atriplex marina Matt. & Atriplex prostrata DC. & poor \\
\hline 6 & Sophera alpin & Cassia sophera L. & poor \\
\hline 6 & Lilius convalius flos Matt. & Convallaria majalis $\mathrm{L}$. & good \\
\hline 6 & Napellus flore ceruleo & Delphinium sp. & poor \\
\hline 6 & Elianthemus indicus G.B. & Helianthus tuberosus L. & bad \\
\hline 6 & / & Paliurus spina-christi Mill. & poor \\
\hline 6 & Ranunculus montanum & Ranunculus sp. & bad \\
\hline 6 & Tanacetum inodorus & Tanacetum corymbosum (L.) Sch. Bip. & good \\
\hline 7 & Alypus monticola thymaelea ? capitulo? & / & bad \\
\hline 7 & Ranunculus globosus & / & good \\
\hline 7 & Myriophyllus Mira triphilla Palustre & / & bad \\
\hline 7 & Napellus folyi laciniatis & / & no sample \\
\hline 7 & $?$ & / & no sample \\
\hline 7 & Horminus Dod. sive Horminus sclarea ?C.B. v. matricala & Horminum pyrenaicum L. & bad \\
\hline 7 & $\begin{array}{l}\text { Amblateum sive orobanche Matt. aut Limodoron Dod. } \\
\text { Caudoleionis, Herbatora }\end{array}$ & Orobanche rapum-genistae Thuill. & good \\
\hline 7 & Minus capitulis seu ?echinodis Bocc. & Paronychia echinatula Chater & good \\
\hline 7 & Trissago sive Camedris? & Teucrium chamaedrys $\mathrm{L}$. & good \\
\hline 8 & Fenum ? & / & good \\
\hline 8 & Ranunculus coriandriflore & / & bad \\
\hline 8 & / & / & no sample \\
\hline 8 & Alchimilla, Stellaria, pes leonis Ted: Sinnaum Franz. pié de leon & Alchemilla sp. & good \\
\hline 8 & Erigerus Dod. sive amellus montanicolus & Aster cf. amellus L. & good \\
\hline 8 & Clynopodium Dios. Pm Acinos Col. & Clinopodium vulgare L. & good \\
\hline 8 & Rapistrum monospermo G.B. & Crambe hispanica L. & good \\
\hline 8 & / & Paliurus spina-christi Mill. & bad \\
\hline 8 & Ranunculus & Ranunculus sp. & good \\
\hline 8 & Teucrium vulgare fruticans? & Teucrium flavum L. & good \\
\hline 9 & Clynopodius? & / & good \\
\hline 9 & Corallina & / & good \\
\hline 9 & Dryopteris nigra dodonei perperas ? & Asplenium adiantum-nigrum L. & good \\
\hline 9 & Cariophyllata montana Ch. & Geum montanum L. & good \\
\hline 9 & Triticum vaccinum $\mathrm{D}$ : & Melampyrum arvense L. & good \\
\hline 9 & Pistactium ? v. fasruchi & Pistacia vera $\mathrm{L}$. & good \\
\hline 9 & Scabiosa minor? & Scabiosa columbaria L. & good \\
\hline 9 & Talictrum? & Thalictrum sp. & good \\
\hline 9 & $\begin{array}{l}\text { Pigamus Dod: ut asservit Dodon : aliqui vocant Rhabarbarus } \\
\text { spurius, Hist: Thalictrus magnus }\end{array}$ & Thalictrum sp. & good \\
\hline 10 & Aconitus lycoctenus $3^{\circ}$ Matt. & Aconitum sp. & good \\
\hline 10 & Ranunculus narcisi flore & Aconitum sp. & good \\
\hline 10 & Flos eius de Anagiris altera & Anagyris foetida $\mathrm{L}$. & poor \\
\hline
\end{tabular}




\begin{tabular}{|c|c|c|c|}
\hline $\begin{array}{l}\text { Herbarium } \\
\text { sheet number }\end{array}$ & $\begin{array}{l}\text { Plant name as indicated } \\
\text { in the Herbarium }\end{array}$ & Current taxonomic identification & $\begin{array}{c}\text { State of } \\
\text { preservation }\end{array}$ \\
\hline 10 & Vulvaria D.C. & Chenopodium vulvaria $\mathrm{L}$. & good \\
\hline 10 & Genzianella minima latifoglia? & Gentiana clusii E. P. Perrier \& Songeon & good \\
\hline 10 & Persica sive Hydropiper M. v. pipi d'acqua & Persicaria maculosa (L.) Gray & bad \\
\hline 10 & Lichnis sylvestris? & Silene sp. & good \\
\hline 11 & Alga membranacea C.B. & / & good \\
\hline 11 & / & / & good \\
\hline 11 & / & / & good \\
\hline 11 & / & / & good \\
\hline 11 & / & / & good \\
\hline 11 & / & / & good \\
\hline 11 & Stella marina & / & good \\
\hline 12 & Chelidonius foliys laciniatis & / & bad \\
\hline 12 & / & / & good \\
\hline 12 & $?$ & / & poor \\
\hline 12 & Gelsominum luteus & / & bad \\
\hline 12 & Cardus stellatus D. & Centaurea calcitrapa L. & poor \\
\hline 12 & Lamnium luteus D.? & Lamium galeobdolon (L.) Crantz & good \\
\hline 12 & / & Scorzonera sp. & good \\
\hline 12 & Talictrum minus & Thalictrum minus L. & good \\
\hline 13 & Centaureum vulg. lacea centauroides & Centaurea sp. & good \\
\hline 13 & $\begin{array}{l}\text { Campanula arvensis Dod. Viola tetragina } \\
\text { onobrichis ?Speculum-veneris }\end{array}$ & Falcata legousia (Ten.) Janch. & good \\
\hline 13 & Persicaria? & Polygonum sp. & good \\
\hline 13 & Ocymum valentinus $\mathrm{CB}$. & Stachys ocymastrum (L.) Briq. & good \\
\hline 13 & Urtica maxima canadensis & Urtica sp. & poor \\
\hline 14 & lacea capite spinoso & Centaurea seridis subsp. sonchifolia (L.) Greuter & good \\
\hline 14 & / & Erigeron sp. & good \\
\hline 14 & Ornus ?Fraxinus minor sylvestris C.B. & Fraxinus ornus L. & good \\
\hline 14 & Persicaria & Polygonum sp. & good \\
\hline 14 & Apios Dod. & Bituminaria bituminosa (L.) C. H. Stirt. & good \\
\hline 14 & Salvia angustifolia & Salvia officinalis $\mathrm{L}$. & good \\
\hline 14 & Ocymastrum valentinus & Stachys ocymastrum (L.) Briq. & good \\
\hline 15 & Ononis non spinosa lutea & / & bad \\
\hline 15 & / & / & no sample \\
\hline 15 & / & / & no sample \\
\hline 15 & Clematis flore pleno coeruleo G.B. & Clematis sp. & good \\
\hline 15 & Rubia maxima narbonensis Col & Crucianella maritima L. & poor \\
\hline 15 & Rubia ? Jo Bauh. Mollugo palustris & Galium rotundifolium L. & good \\
\hline 15 & Paliurus ? & Paliurus spina-christi Mill. & poor \\
\hline 15 & Ranunculus flammeus & Ranunculus flammula $\mathrm{L}$. & poor \\
\hline 15 & Rubia maior Mat. & Rubia peregrina L. & good \\
\hline 15 & Molle arbor clusii sive Lentiscus peruvianus & Schinus molle L. & good \\
\hline 16 & Ebulus laciniatus folii Cor. & / & good \\
\hline
\end{tabular}




\begin{tabular}{|c|c|c|c|}
\hline $\begin{array}{l}\text { Herbarium } \\
\text { sheet number }\end{array}$ & $\begin{array}{l}\text { Plant name as indicated } \\
\text { in the Herbarium }\end{array}$ & Current taxonomic identification & $\begin{array}{c}\text { State of } \\
\text { preservation }\end{array}$ \\
\hline 16 & Centaurius minus vulg. Brundolitta & Centaurium erythraea Rafn & good \\
\hline 16 & Circea foli? & Circaea lutetiana L. & good \\
\hline 16 & Melilotus coronaria Ch. & Dorycnium hirsutum (L.) Ser. & good \\
\hline 16 & / & Euphorbia paralias L. & poor \\
\hline 16 & Filipendula & Filipendula vulgaris Moench & poor \\
\hline 16 & Chamedrius thimi folii elianthemus thimifolio $\mathrm{Ch}$. & Fumana thymifolia (L.) Spach ex Webb. & good \\
\hline 16 & Alsine & Minuartia verna (L.) Hiern & good \\
\hline 16 & Paronichia r. Matt. & Polycarpon tetraphyllum (L.) L. & good \\
\hline 17 & Filix ramosa & / & good \\
\hline 17 & / & / & poor \\
\hline 17 & / & / & no sample \\
\hline 17 & Buglossum & Anchusa sp. & good \\
\hline 17 & Pilosella Dod. & Antennaria dioica (L.) Gaertn. & good \\
\hline 17 & Cirsus & Carduus defloratus L. & poor \\
\hline 17 & Limonius minus $\mathrm{Ch}$ & Limonium sp. & good \\
\hline 17 & / & Lythrum junceum Banks \& Sol. & good \\
\hline 17 & Medica marina Lob. & Medicago marina L. & good \\
\hline 17 & Ononis ? Bauh. & Ononis sp. & good \\
\hline 17 & Ranunculus flammeus & Ranunculus flammula $\mathrm{L}$. & good \\
\hline 17 & Citiso & Genista monspessulana (L.) L. A. S. Johnson & good \\
\hline 18 & Ligusticum alterus & Angelica sylvestris $\mathrm{L}$. & good \\
\hline 18 & Erigerus Dod. sive amellus montanicolum & Aster cf. amellus L. & good \\
\hline 18 & Carduus Cirsis species & Carduus defloratus L. & good \\
\hline 18 & Gramen leucanthemum & Cerastium arvense L. & good \\
\hline 18 & / & Dorycnium pentaphyllum Scop. & good \\
\hline 18 & Stecas crispi foli (index)? & Lavandula dentata $\mathrm{L}$. & poor \\
\hline 19 & Pseudo Dictamnus & Ballota pseudodictamnus (L.) Benth. & good \\
\hline 19 & lacea centauroides (index) & Centaurea sp. & poor \\
\hline 19 & Orminus & Horminum pyrenaicum L. & good \\
\hline 19 & Lisimachia? & Melampyrum sp. & good \\
\hline 19 & Linum umbilicatus & Omphalodes linifolia (L.) Moench & good \\
\hline 20 & Atriplex (index) & Atriplex prostrata DC. & good \\
\hline 20 & lacea centauroides & Centaurea sp. & good \\
\hline 20 & / & Inula helenium L. & good \\
\hline 20 & Salvia & Salvia officinalis $L$. & good \\
\hline 20 & Tragopogon flore ?rubense Lob. & Scorzonera undulata subsp. deliciosa (Guss.) Maire & good \\
\hline 20 & Urtica 3 & Urtica dioica L. & good \\
\hline 21 & ? & / & good \\
\hline 21 & Alcea villosa? & Althea hirsuta L. & good \\
\hline 21 & Thlaspi minus quibusdam aliis Alysson minus nasturtius tragi & Alyssum sp. & good \\
\hline 21 & Lisymachia siliquosa & Epilobium lanceolatum Sebast. \& Mauri & good \\
\hline 21 & Epimedius Plinii ? & Epimedium alpinum L. & good \\
\hline 21 & Urtica iners & Lamium garganicum L. & good \\
\hline
\end{tabular}




\begin{tabular}{|c|c|c|c|}
\hline $\begin{array}{l}\text { Herbarium } \\
\text { sheet number }\end{array}$ & $\begin{array}{l}\text { Plant name as indicated } \\
\text { in the Herbarium }\end{array}$ & Current taxonomic identification & $\begin{array}{c}\text { State of } \\
\text { preservation }\end{array}$ \\
\hline 21 & Orchis & Orchis sp. & poor \\
\hline 21 & Frumentus indicus & Zea mays L. & good \\
\hline 22 & Blitum (index) & / & good \\
\hline 22 & $?$ & Arabis turrita $\mathrm{L}$. & good \\
\hline 22 & Acarna foli & Carlina sicula Ten. & good \\
\hline 22 & / & Cistus incanus L. & good \\
\hline 22 & Hedisarus trifillus canadense & Hedysarum canadense L. & good \\
\hline 22 & Triticum vaccinum & Melampyrum arvense L. & good \\
\hline 22 & Plantago & Plantago major L. & good \\
\hline 22 & Tragoriganus & Satureja sp. & good \\
\hline 23 & / & / & good \\
\hline 23 & Colutea scorpioides & Hippocrepis emerus (L.) Lassen subsp. emerus & good \\
\hline 23 & Limonium arvense & Limonium narbonense Mill. & good \\
\hline 23 & Origano & Origanum vulgare L. & good \\
\hline 23 & Bettonica & Stachys officinalis (L.) Trevis. & good \\
\hline 23 & Asclepium vincetoxicum & Vincetoxicum hirundinaria Medik. & good \\
\hline 24 & / & / & poor \\
\hline 24 & Gnaphalium (index) & Antennaria dioica (L.) Gaertn. & good \\
\hline 24 & Vulneraria rustica & Anthyllis vulneraria $\mathrm{L}$. & poor \\
\hline 24 & Canna indica variegata & Arundo donax L. & good \\
\hline 24 & / & Clematis integrifolia $\mathrm{L}$. & good \\
\hline 24 & Meum & Meum athamanticum Jacq. & good \\
\hline 24 & Psilium tenui foli & Plantago sempervirens Crantz & good \\
\hline 24 & Seseli massiliense seu feniculus tortuosus & Seseli tortuosum L. & good \\
\hline 24 & Ptarmica Austriaca seu lacea olea folia & Xeranthemum inapertum (L.) Mill. & good \\
\hline 25 & Absinthium montanum chamemeli flore & Artemisia absinthium L. & bad \\
\hline 25 & Pseudodictamus & Ballota pseudodictamnus (L.) Benth. & good \\
\hline 25 & Pseudocyperus & Carex sp. & good \\
\hline 25 & ? Botrys ambrosioides & Chenopodium murale L. & good \\
\hline 25 & Epimedius Plinii Icones? & Epimedium alpinum L. & good \\
\hline 25 & Quinquefolius candidus & Potentilla sp. & poor \\
\hline 25 & Eptaphillos & Potentilla sp. & good \\
\hline 26 & Blitum Clus. & Amaranthus deflexus L. & good \\
\hline 26 & Tunica sive cariophyllus sylvestris & Dianthus deltoides $\mathrm{L}$. & good \\
\hline 26 & Cariophyllata alpina & Geum montanum L. & good \\
\hline 26 & Urtica iners altera & Lamium sp. & good \\
\hline 26 & Auricola muris & Myosotis sp. & good \\
\hline 26 & Poliganus Mari & Polygonum maritimum L. & good \\
\hline 26 & Apios arachioides & Bituminaria bituminosa (L.) C. H. Stirt. & good \\
\hline 26 & Seseli massiliense folii feniculum sive feniculus tortuosus & Seseli tortuosum L. & good \\
\hline 26 & Lagopus & Trifolium arvense L. & good \\
\hline 27 & Alissus Mattioli & / & no sample \\
\hline 27 & Acer minor & Acer campestre L. & good \\
\hline
\end{tabular}




\begin{tabular}{|c|c|c|c|}
\hline $\begin{array}{l}\text { Herbarium } \\
\text { sheet number }\end{array}$ & $\begin{array}{l}\text { Plant name as indicated } \\
\text { in the Herbarium }\end{array}$ & Current taxonomic identification & $\begin{array}{c}\text { State of } \\
\text { preservation }\end{array}$ \\
\hline 27 & Daucus & Athamanta sp. & poor \\
\hline 27 & Vitis Indica laciniatis foliis Cor. & Vitis laciniosa L. & good \\
\hline 28 & / & / & no sample \\
\hline 28 & / & / & no sample \\
\hline 28 & Chachalia Clusy & Adenostyles sp. & good \\
\hline 28 & Moli flore sub viridi Boc. & Allium siculum Ucria & good \\
\hline 28 & Moli minimus autunnale G.B. & Allium subvillosum Salzm. ex Schult. \& Schult. f. & poor \\
\hline 29 & / & / & bad \\
\hline 29 & $?$ & / & no sample \\
\hline 29 & Absintium Ponticum & Artemisia pontica L. & good \\
\hline 29 & Solano Vessicario-Pseudo costus & Physalis alkekengi L. & good \\
\hline 29 & Aria theophrasti Lug. & Sorbus aria (L.) Crantz & good \\
\hline 30 & Enula campana & Inula helenium L. & poor \\
\hline 30 & ?Bulbosa montana & Leonurus cardiaca L. & bad \\
\hline 30 & Acetosella arborea & Rumex lunaria L. & good \\
\hline 31 & Sassifragia venetorus & / & good \\
\hline 31 & Carduus minimum? & Atractylis cancellata L. & poor \\
\hline 31 & Consolida maggiore & Symphytum bulbosum K. F. Schimp. & poor \\
\hline 32 & Sysima purpurea & / & poor \\
\hline 32 & / & / & no sample \\
\hline 32 & Dryopteris nigra Dod. - Adianthus nigrus ch. & Asplenium adiantum-nigrum L. & good \\
\hline 32 & Heliotropius minus tricoccus Clus. et Cas. B. & Chrozophora tinctoria (L.) A. Juss. & good \\
\hline 32 & Tithimalus Paralius Dod. et Matth. nonnulli? & Euphorbia paralias L. & good \\
\hline 32 & Heliotropius maius Dios. Herba diabuli, herba di quagli & Heliotropium europaeum L. & good \\
\hline 33 & $?$ & / & bad \\
\hline 33 & Acacia Aegyptiaca seu spina Egyptiaca Teophras. sic cassia & Acacia sp. & good \\
\hline 33 & Lupulus solitarius Dod. & Humulus lupulus L. & poor \\
\hline 33 & Rubia tinctoria & Rubia tinctorum L. & good \\
\hline 33 & $\begin{array}{l}\text { Gramen cassinus maritimus paniculatus sive spicatus G.B. in } \\
\text { Dod. }\end{array}$ & Sporobolus pungens (Schreb.) Kunth & good \\
\hline 34 & Tithimalus Myrsinites seu ? & / & good \\
\hline 34 & $\begin{array}{l}\text { Chamaelea Alpina ? mezerič quo utantur in officinis et precipue } \\
\text { Panormi }\end{array}$ & Daphne alpina L. & poor \\
\hline 34 & Coniza maior Matt. sive Baccharis monsp. Chab & Inula conyzae (Griess.) DC. & good \\
\hline 34 & Virga aurea? & Solidago virga-aurea L. & poor \\
\hline 34 & Virga aurea altera Lobel. Perperas & Solidago virga-aurea $\mathrm{L}$. & good \\
\hline 34 & Vinca Vinca maculosa & Vinca major L. & good \\
\hline 35 & Angelica & Angelica sylvestris $\mathrm{L}$. & poor \\
\hline 35 & Unedo seu arbutus ?et cerosa marine? & Arbutus unedo L. & good \\
\hline 35 & Linaria species & Linaria purpurea (L.) Mill. & poor \\
\hline 36 & Poliganus arborescens & / & no sample \\
\hline 36 & Trichomanes Adiantum nigra? & Asplenium trichomanes L. & bad \\
\hline 36 & Trichomanes Matth. & Asplenium trichomanes L. & poor \\
\hline 36 & ?Matth. & Blechnum spicant (L.) Roth & good \\
\hline
\end{tabular}




\begin{tabular}{|c|c|c|c|}
\hline $\begin{array}{l}\text { Herbarium } \\
\text { sheet number }\end{array}$ & $\begin{array}{l}\text { Plant name as indicated } \\
\text { in the Herbarium }\end{array}$ & Current taxonomic identification & $\begin{array}{c}\text { State of } \\
\text { preservation }\end{array}$ \\
\hline 36 & Seseleos & Bupleurum fruticosum $\mathrm{L}$. & bad \\
\hline 36 & Poliganus minus Matth. seu herniaria seu herba ? li colli di pulizzi & Herniaria glabra subsp. nebrodensis Nyman & good \\
\hline 37 & / & / & no sample \\
\hline 37 & Balsamita acquatica & Impatiens sp. & poor \\
\hline 37 & Euphragia lutea? & Odontites luteus (L.) Clairv. & good \\
\hline 37 & Euphragia sive Euphrorine aut Saris vera Matth. in Diosc. & Odontites luteus (L.) Clairv. & bad \\
\hline 37 & $\begin{array}{l}\text { Solanus sonniferus 4: Matth. perperas Panormi vulgo } \\
\text { Alchechengi solanus fruticosus bacciferus C. B. Amomus Plinij } \\
\text { obs. Lob. V. spezii }\end{array}$ & Withania somnifera (L.) Dunal & poor \\
\hline 38 & Phyllitis Matth. vulgo lingua cervina et scolopendria & / & no sample \\
\hline 38 & Bellis minor Matt. vulgo primo xiuri & Bellis perennis $\mathrm{L}$. & good \\
\hline 38 & $\begin{array}{l}\text { Nardus Italica seu Lavandula Matth. vulgo spica addosso spagl. } \\
\text { espliego }\end{array}$ & Lavandula angustifolia Mill. & good \\
\hline 38 & $\begin{array}{l}\text { Costus Hortensis seu Mentha Greca et Mentha Sarracenica in } \\
\text { Hatruria Salvia Romana ab alys Herba di Mariae ?Balsamita Dod. } \\
\text { vulgo Mentha Romana }\end{array}$ & Tanacetum balsamita L. & poor \\
\hline 38 & / & Taxus baccata L. & good \\
\hline 39 & $\begin{array}{l}\text { Rosa moschata flore pleno Casp. B. Rosa moschata alba } \\
\text { multiphera Taber: Rosa moschata centifolia dicta }\end{array}$ & Rosa moschata Herrm. & good \\
\hline 40 & Anagyris & Anagyris foetida $\mathrm{L}$. & good \\
\hline 40 & V. rumpi quartara & Iris planifolia (Mill.) Fiori \& Paol. & good \\
\hline 40 & Mirtus latifolia (index) v. muttidda adinaria & Myrtus communis L. & good \\
\hline 41 & Camepythys seu Ajuga vulgo Iva Mosch. monsp. Chab. & Ajuga chamaepitys (L.) Schreb. & good \\
\hline 41 & ? Hissopus & Micromeria greca (L.) Benth. & good \\
\hline 41 & Osmunda reale & Osmunda regalis $\mathrm{L}$. & poor \\
\hline 41 & Verbascus papaveris cornuti foliis G.B. & Verbascum sinuatum L. & poor \\
\hline 42 & Epittimus Matt. & Cuscuta epithymum (L.) L. & good \\
\hline 42 & $\begin{array}{l}\text { Caryophyllus indicus seu Othonna major polyanthos vulgo fiori } \\
\text { di morti }\end{array}$ & Tagetes erecta L. & poor \\
\hline 42 & Tagetes seu flos africanus & Tagetes erecta $\mathrm{L}$. & bad \\
\hline 42 & Tanacetum Matt. & Tanacetum sp. & good \\
\hline 43 & $\begin{array}{l}\text { Pimpinella hircina saxifraga Ch. sive Pimpinella hircina saxifraga } \\
\text { semine parvo diuretico Boc. Desc. }\end{array}$ & / & bad \\
\hline 43 & $\begin{array}{l}\text { Scolimus crisanthemus scolimus Theop. Carduus crisanthemus } \\
\text { Dod. vulgo scoddi? }\end{array}$ & Carduus pycnocephalus L. & good \\
\hline 43 & Crithmus (index) & Crithmum maritimum L. & good \\
\hline 43 & Cichoreus costantinopolitanus Dod. Condrilla tuberosa & Leontodon tuberosum L. & good \\
\hline 44 & Chritmus ? Dod: vulgo maio ? Chrisanthemus & / & good \\
\hline 44 & ? & / & bad \\
\hline 44 & $\begin{array}{l}\text { Halimus Portulaca marina vulgo ? Molochia Arab. } \\
\text { Atriplex marina mauritani }\end{array}$ & Atriplex halimus L. & good \\
\hline 44 & Coronopus minor Stellaria cornucervui & Plantago coronopus L. & good \\
\hline 44 & Salix & Salix sp. & good \\
\hline 45 & Malva rosea & / & bad \\
\hline 45 & Adiantus Albus capillus - veneris & Adiantum capillus-veneris $\mathrm{L}$. & good \\
\hline 45 & Gramen filicius polyanthes & Eragrostis cilianensis (All.) Janch. & good \\
\hline 45 & Leucoius marinus sive crucigerus & Matthiola tricuspidata (L.) R. Br. & poor \\
\hline
\end{tabular}




\begin{tabular}{|c|c|c|c|}
\hline $\begin{array}{l}\text { Herbarium } \\
\text { sheet number }\end{array}$ & $\begin{array}{l}\text { Plant name as indicated } \\
\text { in the Herbarium }\end{array}$ & Current taxonomic identification & $\begin{array}{c}\text { State of } \\
\text { preservation }\end{array}$ \\
\hline 45 & Pimpinella hortensis & Sanguisorba minor Scop. & good \\
\hline 46 & Sabina Tamarici similis Hist: Lug: & / & no sample \\
\hline 46 & Ebano nigro & / & good \\
\hline 46 & Papaver & Glaucium flavum Crantz & good \\
\hline 46 & Sabina cupresso similis & Juniperus cf. sabina L. & poor \\
\hline 46 & Cupressus & Juniperus phoenicea L. & good \\
\hline 47 & Stratiotes, Millefolia minor Matth. & Achillea sp. & good \\
\hline 47 & Kali gerina seu Soda & Salicornia sp. & good \\
\hline 48 & Serpentaria minima ?Cup. & Biarum tenuifolium (L.) Schott & bad \\
\hline 48 & Cineraria sive lacobea marina Jo. B. & Senecio sp. & good \\
\hline 48 & Polimius Dod. seu Been Albo Monsp. & Silene sp. & good \\
\hline 48 & Vitex Matth. Agnus castus Gatillo casto Hispani & Vitex agnus - castus L. & poor \\
\hline 49 & Linaria & Antirrhinum majus $\mathrm{L}$. & poor \\
\hline 49 & Geranius ? & Geranium sp. & good \\
\hline 49 & Narcissus ?albus & Narcissus tazetta L. & good \\
\hline 49 & Ribes vulgares & Ribes sp. & bad \\
\hline 49 & Hedypnois Valer: Rostrus porcinus officine Dentes leonis? & Sonchus oleraceus L. & poor \\
\hline 50 & Hipp: cavallo marinum & / & poor \\
\hline 50 & Stella marina echinacea & / & good \\
\hline 50 & $\begin{array}{l}\text { Coralloides fruticosa Planta marina ?corallina fruticosa Gesner. } \\
\text { iuncus sapideus anguil. Corallina alba Tabern lithophiton } \\
\text { marinus albicans Jo. B. }\end{array}$ & / & good \\
\hline 50 & Vessicaria marina? & Asplenium scolopendrium L. & bad \\
\hline 51 & Rapontico (index) & / & bad \\
\hline 51 & Cerasus amara Mahaleb ? & Prunus mahaleb L. & poor \\
\hline 51 & Horminus major & Salvia sp. & bad \\
\hline 51 & Polium p.m Matth. & Teucrium polium L. & good \\
\hline 52 & Althea & Althaea officinalis $L$. & poor \\
\hline 52 & Filipendula & Filipendula vulgaris Moench & poor \\
\hline 52 & Limonio & Limonium narbonense Mill. & poor \\
\hline 53 & Paronichia Matt. Ruta Muraria Adiantus albus & Asplenium ruta-muraria $\mathrm{L}$. & good \\
\hline 53 & Hippomarathrum siculus? & Cachrys sicula L. & good \\
\hline 53 & Cyperus Mat: & Cyperus sp. & good \\
\hline 54 & Lotus arbor M. siculi caccama & Celtis australis L. & poor \\
\hline 55 & Verbena nodiflora? & Phyla nodiflora (L.) Greene & poor \\
\hline 55 & Sandolina cretica ? Lotus ruber siliqua angulosa B. Pin. & Lotus tetragonolobus L. & good \\
\hline 56 & Colutea scorpioides ? Scorpioides arborescens J. B. & Hippocrepis emerus (L.) Lassen subsp. emerus & good \\
\hline 56 & Peonia mas & Paeonia mascula (L.) Mill. & good \\
\hline 56 & Geranium moscatum & Pelargonium sp. & good \\
\hline 57 a & Sic. Crozza di Morti & Antirrhinum majus $\mathrm{L}$. & poor \\
\hline 57 a & Pimpinella sive Sanguisorbia & Sanguisorba minor Scop. & good \\
\hline $57 \mathrm{~b}$ & Thlaspi biscutatus Hieracifolius & Biscutella lyrata L. & good \\
\hline $57 \mathrm{~b}$ & Blattaria Matt. & Verbascum blattaria L. & good \\
\hline 58 & Abies minor Dod. & Abies alba Mill. & good \\
\hline
\end{tabular}




\begin{tabular}{|c|c|c|c|}
\hline $\begin{array}{l}\text { Herbarium } \\
\text { sheet number }\end{array}$ & $\begin{array}{l}\text { Plant name as indicated } \\
\text { in the Herbarium }\end{array}$ & Current taxonomic identification & $\begin{array}{c}\text { State of } \\
\text { preservation }\end{array}$ \\
\hline 58 & Anemone tuberosa radice Bulbo Castano radice Ch. e C.B. sive? & Anemone apennina L. & poor \\
\hline 58 & Pimpinella spinosa & Sarcopoterium spinosum (L.) Spach & good \\
\hline 59 & Gramen maximus Dalec. & Cyperus capitatus Vand. & good \\
\hline 59 & Verbascum salvifolium G. B. 4. m Matth. & Phlomis fruticosa L. & good \\
\hline 59 & Matricale seu Horminus & Stachys sp. & poor \\
\hline 60 & Asteratticus & Aster cf. amellus L. & good \\
\hline 60 & Lathyrus angustifolia Ch. & Lathyrus sp. & good \\
\hline 61 & Cytisus Hispanicus $2^{\circ}$ Clus. & Cytisus villosus Pourr. & good \\
\hline 61 & Helychrysum orientale C. B. Pin. & Phagnalon saxatile (L.) Cass. & good \\
\hline 62 & / & / & bad \\
\hline 62 & $\begin{array}{l}\text { Scabiosa argentea ? Jo: Bauh. Stoebe salmantica } \\
\text { minor Clus. E Lob. }\end{array}$ & Centaurea sp. & good \\
\hline 62 & Milium solis & Lithospermum sp. & poor \\
\hline 62 & Scabiosa arborea Alp. & Lomelosia cretica (L.) Greuter \& Burdet & good \\
\hline 63 & Cistus Ledon alterus Clus. & Cistus monspeliensis L. & good \\
\hline 63 & Gladiolus unofloris Mor: & Gladiolus sp. & good \\
\hline 64 & / & Camphorosma monspeliaca $\mathrm{L}$. & good \\
\hline 64 & Gladiolus binis flores ordinibus cinctus Lob. & Gladiolus sp. & good \\
\hline 64 & Plantago alopecuros & Plantago lagopus L. & good \\
\hline 64 & Hedypnois Monspelliensis sive? & Sonchus oleraceus L. & good \\
\hline 65 & Consolida regalis flore ceruleo & Consolida sp. & good \\
\hline 65 & Consolida regalis flore albo & Consolida sp. & good \\
\hline 66 & Cyanus minor Matt. Cyanus segetus & Cyanus segetum Hill & good \\
\hline 66 & Cyanus & Cyanus segetum Hill & good \\
\hline 67 & Carduus stellatus sive Myacanthos Teoph. Lugd. & Centaurea calcitrapa L. & good \\
\hline 67 & Nigella sive Melanthium vel Gith. & Nigella damascena $\mathrm{L}$. & good \\
\hline 67 & Odontites Plin. Bella Margarita Siculi & Viola arvensis Murray & good \\
\hline 68 & Consolida Regalis fl. violaceo & Consolida sp. & good \\
\hline 68 & Ricinus Matt. Sic. Erba Caruana & Ricinus communis $\mathrm{L}$. & good \\
\hline 68 & Tussilago sive farfara officinarus & Tussilago farfara $\mathrm{L}$. & good \\
\hline 69 & Thlaspi arborescens Prosp. Alp. fiore bianco siculis & Iberis semperflorens L. & good \\
\hline 69 & Linus & Linum usitatissimum L. & good \\
\hline 69 & Tragopogon seu Barba Hirci Siciliano Percia Cannelli & Tragopogon crocifolius L. & good \\
\hline 70 & Amomus & / & poor \\
\hline 70 & Angelica acquatica sicula & Angelica sylvestris L. & poor \\
\hline 70 & Pulmonaria vera & Pulmonaria sp. & poor \\
\hline 70 & Sanguis draconis & Rumex sp. & good \\
\hline 70 & Tussilago seu farfara sic. Unghia cavaddina & Tussilago farfara $\mathrm{L}$. & poor \\
\hline 70 & Viola nigra & Viola cf. odorata L. & good \\
\hline 71 & Cherefolium & Anthriscus sp. & good \\
\hline 71 & Etiopius Matt. & Salvia aethiopis L. & good \\
\hline 71 & Saponaria & Saponaria officinalis L. & poor \\
\hline 72 & Ruta muraria & Asplenium ruta-muraria $\mathrm{L}$. & good \\
\hline
\end{tabular}




\begin{tabular}{|c|c|c|c|}
\hline $\begin{array}{l}\text { Herbarium } \\
\text { sheet number }\end{array}$ & $\begin{array}{l}\text { Plant name as indicated } \\
\text { in the Herbarium }\end{array}$ & Current taxonomic identification & $\begin{array}{c}\text { State of } \\
\text { preservation }\end{array}$ \\
\hline 72 & Laurus imperiale & Laurus sp. & poor \\
\hline 72 & Erba turca (index) & Spergularia rubra (L.) J. Presl \& C. Presl & poor \\
\hline 72 & Bettonica & Stachys officinalis (L.) Trevis. & good \\
\hline 73 & Celidoniam majus Matt. vulgo Celidonia Hirundinaria & Chelidonium majus L. & poor \\
\hline 73 & Primula veris seu Conterba Sic. (index) & Primula acaulis (L.) L. & poor \\
\hline 73 & Solano racemoso & Solanum sp. & good \\
\hline 73 & Satureja Sic. riganeddu & Thymbra capitata (L.) Cav. & good \\
\hline 74 & Alchimilla seu Erba stellaria (index) & Alchemilla sp. & good \\
\hline 74 & Cariophillata & Geum montanum L. & poor \\
\hline 74 & Ligustrum & Ligustrum vulgare $\mathrm{L}$. & poor \\
\hline 74 & Geranio ? & Pelargonium sp. & poor \\
\hline 75 & / & / & poor \\
\hline 75 & / & / & no sample \\
\hline 75 & Limonio & Citrus limon (L.) Burm. f. & poor \\
\hline 75 & Meliante & Sanguisorba minor Scop. & poor \\
\hline 76 & Drago Cefalo & / & bad \\
\hline 76 & Arundo indica (index) & Arundo donax L. & bad \\
\hline 76 & Galega Ruta Caprarica (index) & Galega officinalis L. & poor \\
\hline 77 & Dragoriganum & / & poor \\
\hline 77 & Stecas Erba di Pal. (index) & Lavandula stoechas L. & poor \\
\hline 77 & Rutha hortensis (index) & Ruta chalepensis L. & poor \\
\hline 78 & Pentiphyllus major & / & good \\
\hline 78 & Fumaria & Fumaria sp. & good \\
\hline 78 & Serpillum & Thymus serpyllum L. & good \\
\hline 79 & Aquilegia & Aquilegia vulgaris $\mathrm{L}$. & poor \\
\hline 79 & Filix mas & Dryopteris filix-mas (L.) Schott & poor \\
\hline 80 & / & Anthemis sp. & good \\
\hline 80 & Geranium alterum pede Columbino & Geranium pyrenaicum Burm. f. & poor \\
\hline 81 & Cicerbita & / & good \\
\hline 81 & Siderite ?seu Strigarella & Lycopus europaeus L. & poor \\
\hline 81 & Ormino minore (index) & Stachys sp. & poor \\
\hline 82 & Nummularia & Lysimachia nummularia L. & poor \\
\hline 82 & $?$ & Rubus canescens DC. & poor \\
\hline 82 & Adianthum & Thalictrum aquilegiifolium L. & good \\
\hline 83 & Brionia & Bryonia cretica subsp. dioica (Jacq.) Tutin & poor \\
\hline 83 & Cariophillata & Geum urbanum L. & good \\
\hline 84 & Ononis sic resta bovis & Ononis sp. & good \\
\hline 84 & ? passerina (index) & Polygonum maritimum L. & bad \\
\hline 84 & Solanum pomiferum & Solanum linnaeanum Hepper \& P.-M. L. Jaeger & good \\
\hline 84 & Maro Cortuso & Thymus serpyllum L. & good \\
\hline 84 & Veronica femmina & Veronica anagallis-aquatica $\mathrm{L}$. & good \\
\hline 84 & Veronica maschio & Veronica sp. & poor \\
\hline 85 & luda & Cercis siliquastrum L. & poor \\
\hline
\end{tabular}




\begin{tabular}{|c|c|c|c|}
\hline $\begin{array}{l}\text { Herbarium } \\
\text { sheet number }\end{array}$ & $\begin{array}{l}\text { Plant name as indicated } \\
\text { in the Herbarium }\end{array}$ & Current taxonomic identification & $\begin{array}{c}\text { State of } \\
\text { preservation }\end{array}$ \\
\hline 85 & Staphisagria sic. ? & Delphinium staphysagria L. & poor \\
\hline 85 & Tormentilla & Geranium versicolor L. & poor \\
\hline 86 & Acanthus Brancaorsina (index) & Acanthus mollis L. subsp. mollis & poor \\
\hline 86 & Cicer syl. Anonis trifoliis affinis Chab. & Ononis natrix subsp. ramosissima (Desf.) Batt. & good \\
\hline 87 & Gramen tremuleus majus phallaris ? B. Pin. & Briza maxima $\mathrm{L}$. & good \\
\hline 87 & Medica follicolo spinoso Lob. & Medicago polymorpha L. & good \\
\hline 87 & Trifolius pratense Trifolius lupulinus femina Ch. & Trifolium badium Schreb. & good \\
\hline 88 & Cistus ladon & Cistus monspeliensis L. & good \\
\hline 88 & Erica purpura ? flore Juniperi foli Lob. & Erica multiflora L. & good \\
\hline 88 & $\begin{array}{l}\text { Hedisarus ? clypeatus sive Helenius Aegyptium Lob. Sulla } \\
\text { Arabum Sulla siculis }\end{array}$ & Hedysarum coronarium L. & poor \\
\hline 88 & Linaria? & Linaria purpurea (L.) Mill. & good \\
\hline 89 & / & / & poor \\
\hline 89 & Narcissus & Narcissus poeticus L. & good \\
\hline 89 & Lichnis sylvestris & Silene dioica (L.) Clairv. & good \\
\hline 90 & Apocynum amplexicaule Lob. sive Periploca repens & Cynanchum acutum L. & good \\
\hline 90 & Crucem syl. Herbariorus Lob. & Pisum sativum L. & good \\
\hline 90 & Scandix Pecten Veneris & Scandix pecten-veneris $\mathrm{L}$. & good \\
\hline 91 & Buglossum verum Lugd. & Borago officinalis L. & good \\
\hline 91 & Cucummis sylvestris sive Asininus & Ecballium elaterium (L.) A. Rich. & poor \\
\hline 91 & Petasites Lob. & Petasites pyrenaicus (L.) G. López & poor \\
\hline 92 & Pastinaca sativa domestica Matth. & Pastinaca sativa L. & good \\
\hline 92 & Circea monspellientium Vitis sylvestris Matt. dulcis? & Solanum dulcamara L. & good \\
\hline 93 & Curriola & / & bad \\
\hline 93 & Mille folium major & Achillea millefolium L. & poor \\
\hline 93 & Perforata seu Petasidis & Adenostyles sp. & bad \\
\hline 94 & Paludapium sic. Appio vigo & Apium sp. & good \\
\hline 94 & Scrophularia peregrina & Scrophularia peregrina $\mathrm{L}$. & good \\
\hline 95 & Panax (index) & / & poor \\
\hline 95 & Ammi Cord. & Ammi sp. & poor \\
\hline 95 & Peucedano ? la seta & Gomphocarpus fruticosus (L.) W. T. Aiton & poor \\
\hline 95 & Lupulus solictarius & Humulus lupulus L. & bad \\
\hline 96 & Cicuta Mat. & Conium maculatum L. & good \\
\hline 96 & Dictamnus Creticus Mat. & Origanum dictamnus L. & good \\
\hline 96 & Oxilapatum sic. Lapazzu & Rumex obtusifolius L. & bad \\
\hline 96 & Orminus sclarea dictus & Salvia sclarea L. & bad \\
\hline 97 & $\begin{array}{l}\text { Cneorum argenteum Mat. Herba cattiva siculis cneorus folio } \\
\text { argenteo molli G. B. doricnius ex Sicilia? }\end{array}$ & Convolvulus cneorum L. & good \\
\hline 97 & Cariophillata M. Herba benedicta & Geum montanum L. & poor \\
\hline 97 & Melissa melecophyllus Mat. & Melissa sp. & good \\
\hline 97 & Rheseda candida cauda equina siculi cauda d'agneddu & Reseda alba L. & poor \\
\hline 98 & Pilosella & Hieracium sp. & poor \\
\hline 98 & Pigamus Dod. Thalictrus majus seu Politrico & Thalictrum aquilegifolium L. & good \\
\hline 99 & Fragaria il suo frutto frauli & Fragaria vesca L. & good \\
\hline
\end{tabular}




\begin{tabular}{|c|c|c|c|}
\hline $\begin{array}{l}\text { Herbarium } \\
\text { sheet number }\end{array}$ & $\begin{array}{l}\text { Plant name as indicated } \\
\text { in the Herbarium }\end{array}$ & Current taxonomic identification & $\begin{array}{c}\text { State of } \\
\text { preservation }\end{array}$ \\
\hline 99 & Condrillas affinis ?sic. ?Chondrilla ?siculis & Reichardia picroides (L.) Roth & good \\
\hline 99 & Nasturtium Indicus Casp. Siculi fiore di passione & Tropaeolum majus $\mathrm{L}$. & poor \\
\hline 100 & Lathiris sive Cataputia minor Tithymalus latifolius Matt. & Euphorbia sp. & poor \\
\hline 100 & Euphragia latifolia purpurea & Parentucellia latifolia (L.) Caruel & poor \\
\hline 100 & Arundo & Phragmites australis (Cav.) Steud. & good \\
\hline 101 & $\begin{array}{l}\text { Apocynus folio rotundiore flore ex albo pallescente G.B. Periploca } \\
\text { graeca foliis latioribus hederaceis Lobelly Cynocrambe Galeni et } \\
\text { cynomoris? periploca repens Caes. Brassica canina }\end{array}$ & Cynanchum acutum L. & good \\
\hline 102 & Anthyllis leguminosa Belgarum & Anthyllis vulneraria $\mathrm{L}$. & good \\
\hline 102 & Stachys spuria Flandrorus Lob. & Sideritis sp. & good \\
\hline 103 & Camphorota Monspelliensis Lob. & Camphorosma monspeliaca $\mathrm{L}$. & good \\
\hline 103 & Nicotiana seu Tabacco & Nicotiana tabacum L. & poor \\
\hline 103 & Thanacetus luteus Athanasia & Tanacetum vulgare $\mathrm{L}$. & good \\
\hline 104 & Scabiosa argentea squammata Jo. Bauh. & Centaurea sp. & good \\
\hline 104 & Stichas citrina latifolia Ch. vulg. Fiore di Messina & Helichrysum pendulum (C. Presl) C. Presl & good \\
\hline 104 & Ageratum ferulae (index) & Lonas annua (L.) Vines \& Druce & good \\
\hline 104 & Chamaedris assurgens Ch. & Teucrium chamaedrys $L$. & good \\
\hline 105 & $?$ & / & poor \\
\hline 105 & Horminum denticulatum & Salvia pratensis L. subsp. pratensis & poor \\
\hline 105 & Scrofularia? & Scrophularia nodosa L. & poor \\
\hline 106 & Peucedanum & Peucedanum sp. & good \\
\hline 106 & Alsina veronica folio G.B. & Veronica arvensis L. & good \\
\hline 106 & Vinca pervinca clematidis & Vinca major L. & bad \\
\hline 107 & Napellus verus Napellus flore ceruleo C.B. & / & good \\
\hline 107 & Brassica marina Soldanella Ch. Mabathamaticon & Calystegia soldanella (L.) Roem. \& Schult. & good \\
\hline 108 & Consolida media Matth. ? & Ajuga orientalis L. & good \\
\hline 108 & Chamaeleon exigus ?carduncellus? & Carthamus pinnatus Desf. & poor \\
\hline 108 & Polium Mattioli & Teucrium polium L. & good \\
\hline 108 & $\begin{array}{l}\text { Serpillum Tragoriganum Clus.vulgare repens H. vide } \\
\text { descriptiones eiusde }\end{array}$ & Thymus striatus Vahl & poor \\
\hline 109 & Dictamnom album Matt. sive fraxinella & Dictamnus albus L. & poor \\
\hline 109 & Tithymalus paralius M. & Euphorbia paralias L. & good \\
\hline 109 & Aphaca vera Dod. Aphaca ? Chab. & Lathyrus aphaca L. & good \\
\hline 109 & Thalictrus majus (index) & Thalictrum sp. & poor \\
\hline 110 & Viola matronalis ?Dioscor. Clinopodius Mat. Acinos? & Acinos alpinus (L.) Moench & poor \\
\hline 110 & Auricula muris ? sive Olosteum tomentosum & Cerastium tomentosum L. & good \\
\hline 110 & Periclymenus seu mater silve ? caprifoglio? & Lonicera etrusca Santi & poor \\
\hline 110 & Solanus magnus virginianus rubrum Park sive americanus? & Phytolacca americana L. & poor \\
\hline 111 & Costus hortensis sive Mentha Greca & Tanacetum balsamita L. & poor \\
\hline 111 & Aconitus lycostonus fl. Delphinius Lob. & Delphinium fissum Waldst. \& Kit. & good \\
\hline 111 & Symphitum nigrus tuberosus siculis & Symphytum tuberosum L. & poor \\
\hline 112 & Elleborus niger (index) & Helleborus sp. & poor \\
\hline 112 & Mentha? & Mentha spicata L. & poor \\
\hline 112 & Phyllitis lingua cervina & Salsola oppositifolia Desf. & poor \\
\hline
\end{tabular}




\begin{tabular}{|c|c|c|c|}
\hline $\begin{array}{l}\text { Herbarium } \\
\text { sheet number }\end{array}$ & $\begin{array}{l}\text { Plant name as indicated } \\
\text { in the Herbarium }\end{array}$ & Current taxonomic identification & $\begin{array}{l}\text { State of } \\
\text { preservation }\end{array}$ \\
\hline 112 & Thalictrus minus & Thalictrum minus $\mathrm{L}$. & poor \\
\hline 113 & Tragacantha Matth. & Astracantha sicula (Raf.) Greuter & good \\
\hline 113 & Petroselinum macedonium & Bubon macedonicum L. & good \\
\hline 113 & $\begin{array}{l}\text { Rhamnus catharticus spina ?cervi spina spina merlo spina } \\
\text { Quercia }\end{array}$ & Rhamnus catharticus L. & bad \\
\hline 114 & Personata major sive Bardana & Arctium minus (Hill) Bernh. & poor \\
\hline 114 & Abrotanus & Artemisia absinthium L. & poor \\
\hline 114 & Rapistrum flore albo cruceifoliis & Diplotaxis erucoides (L.) DC. & bad \\
\hline 114 & Ebulus sive sambucus humilis & Sambucus ebulus $L$. & good \\
\hline 115 & Mentha gattaria sive Herba gatta & Nepeta cataria L. & good \\
\hline 115 & Paronichia rutacea folia Lob. & Saxifraga tridactylites $\mathrm{L}$. & good \\
\hline 115 & $?$ & Jacobaea ambigua (Biv.) Pelser \& Veldkamp & good \\
\hline 115 & Smyrnium creticus Lob. v. lisciandreddu & $\begin{array}{l}\text { Smyrnium perfoliatum subsp. rotundifolium (Mill.) } \\
\text { Bonnier \& Layens }\end{array}$ & poor \\
\hline 116 & Olea boemica & Elaeagnus angustifolia L. & good \\
\hline 116 & Lathyrus latifolia & Lathyrus ochrus (L.) DC. & poor \\
\hline 116 & Mercurialis mas. & Mercurialis annua L. & poor \\
\hline 117 & Chamemelus inodorus sive cotula no fetida & Chamaemelum fuscatum (Brot.) Vasc. & poor \\
\hline 117 & Locusta ? Valleria peregrina pratensis & Fedia cornucopiae (L.) Gaertn. & poor \\
\hline 117 & / & Lotus cytisoides L. & good \\
\hline 117 & Melilotus italica folliculis rotundis & Melilotus italicus (L.) Lam. & poor \\
\hline 118 & Geranius ? longissimo & Erodium moschatum (L.) L'Hér. & poor \\
\hline 118 & Theucrius Beticus & Teucrium fruticans $L$. & good \\
\hline 118 & Verbena & Verbena officinalis $L$. & good \\
\hline 119 & Cotula fetida & Anthemis cotula L. & good \\
\hline 119 & Rubeola arvensis & Asperula arvensis $\mathrm{L}$. & good \\
\hline 119 & / & Cerastium sp. & good \\
\hline 119 & Alsine maritima Neapolitana & Spergularia marina (L.) Griseb. & good \\
\hline 120 & Beta cretica semine aculeato Gasp. B. & Emex spinosa (L.) Campd. & poor \\
\hline 120 & Leodorus marinus & Erysimum cheiri (L.) Crantz & poor \\
\hline 120 & Pisjllius & Plantago afra L. & good \\
\hline 120 & $\begin{array}{l}\text { Valerianella scabiosa ?vulgo cugni moddi erba molle Lazzucheddi } \\
\text { Barba di Monici }\end{array}$ & Valerianella sp. & good \\
\hline 121 & Gallius flore rubro & Asperula aristata L. f. & good \\
\hline 121 & Trissago apulafolii Colum. Euphragia pratensis purp. Iatifolia & Bellardia trixago (L.) All. & good \\
\hline 121 & Pilosella Auricula muris Chab. & Cerastium sp. & good \\
\hline 121 & $\begin{array}{l}\text { Condrilla pusilla marina lutea bulbosa an Diosc. Radice } \\
\text { rotunda orbiculata strongula Theophrasti Cichorius, et } \\
\text { an Hemorrhoidalis Cicorius strumosus Myconis Cichorius } \\
\text { bulbosus Dalesc. }\end{array}$ & Sonchus bulbosus (L.) N. Kilian \& Greuter & good \\
\hline 122 & Perfoliata Dodonei & / & poor \\
\hline 123 & Camedaphnoides Auricularia ?Chab. speculum - veneris & Legousia speculum-veneris (L.) Chaix & poor \\
\hline 123 & Perfoliata siliquosa vel Brassica campestris flore purpureo B. & Moricandia arvensis (L.) DC. & good \\
\hline 123 & Succisa Matt. vulg. Morsus Deaboli & Succisa pratensis Moench & poor \\
\hline 124 & Campanula ocimifol. hirsuta flore pendulo Bocconi Viola mariana & Campanula dichotoma L. & bad \\
\hline
\end{tabular}




\begin{tabular}{|c|c|c|c|}
\hline $\begin{array}{l}\text { Herbarium } \\
\text { sheet number }\end{array}$ & $\begin{array}{l}\text { Plant name as indicated } \\
\text { in the Herbarium }\end{array}$ & Current taxonomic identification & $\begin{array}{l}\text { State of } \\
\text { preservation }\end{array}$ \\
\hline 124 & Cystus Hyspanicus Clus. & Cytisus villosus Pourr. & bad \\
\hline 124 & Origanus onitis & Origanum onites L. & good \\
\hline 124 & Gramen trematus minimus & Ochlopoa annua (L.) H. Scholz & good \\
\hline 125 & Lepidius Pauli et Plinii & / & poor \\
\hline 125 & Centaurius majus vel Rhaponticus perperas & Centaurea sp. & poor \\
\hline 125 & lacea oleafolia Ptarmica altera Matt. Sternutamentaria? & Xeranthemum inapertum (L.) Mill. & good \\
\hline 126 & Cerifolius & Anthriscus cerefolium (L.) Hoffm. & poor \\
\hline 126 & Aristolochia? & Aristolochia sicula Tineo & poor \\
\hline 126 & Genistella & Genista gasparrini (Guss.) C. Presl & good \\
\hline 126 & / & Geranium sp. & good \\
\hline 126 & Sanicula? & Sanicula europaea $\mathrm{L}$. & good \\
\hline 127 & Poliganus niveus & Paronychia argentea Lam. & good \\
\hline 127 & Viscus quercinus (index) & Viscum album L. & poor \\
\hline 128 & ? Siculi caulicettu o Lippu di ? & / & good \\
\hline 128 & Oenanthe sive filipendula? & Oenanthe pimpinelloides L. & poor \\
\hline 128 & / & Xeranthemum inapertum (L.) Mill. & good \\
\hline 129 & ? agrimoniodes & Aremonia agrimonioides (L.) DC. & good \\
\hline 129 & $\begin{array}{l}\text { Phallaris minimus seu Gramen tremulus minimus C.B. Theatri } \\
\text { Botanicae }\end{array}$ & Briza minor L. & good \\
\hline 129 & lacobea? & Senecio sp. & good \\
\hline 130 & / & / & bad \\
\hline 130 & $\begin{array}{l}\text { Echium syl. minus Bocc. Buglossum minimum echiy facie flore } \\
\text { rubense Lob. echioides alba parva Col. Amphybia Caes. }\end{array}$ & Buglossoides arvensis (L.) I. M. Johnst. & poor \\
\hline 130 & $\begin{array}{l}\text { Hedera humilis sive chamecissus Corona terrae, Hedera } \\
\text { terrestris? Cordi }\end{array}$ & Lamium garganicum L. & poor \\
\hline 131 & Artemisia vera Mat. & Artemisia verlotiorum Lamotte & good \\
\hline 131 & Lotus odora angustifolia Lotus urbana Cos. & Melilotus infestus Guss. & good \\
\hline 131 & $\begin{array}{l}\text { Acetosa romana ocymifolio G.B. Acetosa minor ocymifolio } \\
\text { neapolitana Col. }\end{array}$ & Rumex bucephalophorus L. & poor \\
\hline 132 & Viorna vulgi Lob. Clematis $3^{\circ}$ Matth. & Clematis vitalba $\mathrm{L}$. & poor \\
\hline 132 & Cichorius? & Leontodon tuberosum L. & poor \\
\hline 132 & Trifolium corniculatus & Lotus corniculatus L. & good \\
\hline 133 & Phalliris seu gramen tremulus & Briza maxima $\mathrm{L}$. & good \\
\hline 133 & Medica folliculo spinoso & Medicago intertexta (L.) Mill. & poor \\
\hline 133 & Scutellaria cortus, Cassida Col. & Scutellaria columnae All. & good \\
\hline 133 & luiuba syl. Caesal. & Ziziphus lotus (L.) Lam. & good \\
\hline 134 & Geranium fuschum Lob. & Geranium versicolor $\mathrm{L}$. & poor \\
\hline 134 & Medica folliculo cochleato & Medicago orbicularis (L.) Bartal. & good \\
\hline 134 & Cicer sylvaticum Theriacaria & Ononis natrix L. subsp. natrix & good \\
\hline 135 & Flos adonis Lob. ? Rinedda di Ventu cu xiuri russu & Adonis annua L. subsp. annua & good \\
\hline 135 & Gramen avenaceum & Anisantha madritensis (L.) Nevski & good \\
\hline 135 & Gramen Sorghi effigie & Poa sp. & good \\
\hline 136 & Lychnis & Agrostemma githago L. & poor \\
\hline 136 & Cariophyllata & Geum urbanum L. & poor \\
\hline 136 & Herba venti vel Sideritis Monspelliensius Lobell. & Phlomis herba-venti L. & good \\
\hline
\end{tabular}


Herbarium sheet number
Plant name as indicated

in the Herbarium
Current taxonomic identification

Euphorbia chamaesyce L.

Medicago turbinata (L.) All.

Lavandula sp.

Melittis melissophyllum subsp. albida (Guss.) P. W. Ball

Sisymbrium irio L.

/

139 Thlapsi bellidi folio

139 Auricula muris pulchro flore rosa

139 Ipericon nero

139 Git, sive Melanthium vel nigella

140 Draba $2^{\circ}$ Clus.

$140 \quad$ Lathyrus

140 Anonis lutea no spinosa repens folio lucido serrato Bocconi

141 Cerinthe maior Lob. siculij sucameli

141 /

141 Pentaphyllum erectum Fuchs.

142 Chamemoly an Moly Diosc. Col.

142 Saxifraga?

142 Horminus syl. Fuch.

142 Scabiosa Sic. Herba di Cavaleri

143 Aparine Matth.

143 Geranium Robertianus

143 Herba gatta

144 /

144 Adianthum nigrus Chab. Driopteris nigra Dod.

144 Coluthea scorpioides

144 Linaria Valentina 3philla Clus.

145 Gramen cyperoides

145 Styrax Matth.

145 Talictrum minus

146 Crisanthemum

146 Lilium convalium

146 Euphragia pratense purpurea latifolia Columnae

146 Verbascum

147 Althea arborea Olbia in Gallo Provincia Lobel.

147 Anagallis acquatica Dodonei Becabunga Germanius

$147 \quad$ Vicia

148 Bardana officinalis ? lappola maggiore

148 Rosa sinensis arbor?

148 Sorbus

148 Lappa inversa seu Bardana minor ?strumaria ?

149 Lithospermum ?

149 Saponaria vera
Cerastium tomentosum L. Hypericum perfoliatum L. poor

Nigella damascena $\mathrm{L}$.

Arabis alpina subsp. caucasica (Willd.) Briq. good

Lathyrus gorgonii Parl. good

Ononis oligophylla Ten. good

Cerinthe major L. good

Asplenium ceterach L. good

Potentilla sp. good

Allium chamaemoly L. poor

Micromeria juliana (L.) Benth. ex Rchb. good

Salvia verbenaca $L$. poor

Scabiosa columbaria L. good

Galium aparine L. good

Geranium robertianum L. good

Nepeta cataria L. poor

/ poor

Asplenium adiantum-nigrum L. good

Hippocrepis emerus (L.) Lassen subsp. emerus good

Linaria reflexa (L.) Desf. good

Carex cuprina (Heuff.) A. Kern. good

Styrax officinalis L. poor

Vitex agnus-castus L. poor

Glebionis segetum (L.) Fourr. poor

Convallaria majalis L. poor

Parentucellia latifolia (L.) Caruel good

Verbascum thapsus L. poor

Malva arborea (L.) Webb \& Berthel. good

Veronica anagallis-aquatica $\mathrm{L}$ good

Vicia melanops Sibth. \& Sm. good

Arctium minus (Hill) Bernh. poor

Hibiscus rosa-sinensis L. bad

Sorbus aucuparia L. poor

Xanthium strumarium L. poor

Coix lacryma-jobi L. good

Saponaria officinalis L. good 


\begin{tabular}{|c|c|c|c|}
\hline $\begin{array}{l}\text { Herbarium } \\
\text { sheet number }\end{array}$ & $\begin{array}{l}\text { Plant name as indicated } \\
\text { in the Herbarium }\end{array}$ & Current taxonomic identification & $\begin{array}{c}\text { State of } \\
\text { preservation }\end{array}$ \\
\hline 149 & $\begin{array}{l}\text { Hedera spinosa seu smilax aspera Ital. Rovo cervi vulgo Salsa } \\
\text { siciliana }\end{array}$ & Smilax aspera $\mathrm{L}$. & good \\
\hline 150 & Aloe ex America Dod. Folio in obovatus absente Casp. B. & / & poor \\
\hline 150 & Asparagus silvestris tenuissimis foliis & Asparagus albus L. & good \\
\hline 150 & $\begin{array}{l}\text { Asparagus hortensis Dod. Asparagus sativa Casp. B. vulgo } \\
\text { sparaci }\end{array}$ & Asparagus officinalis L. & good \\
\hline 151 & Arisarus prius Matt. Aris Plin. & Arisarum vulgare O. Targ. Tozz. & good \\
\hline 151 & Sticas citrina ? G.B. & Helichrysum sp. & good \\
\hline 151 & Abrotanum femina Matth. & Santolina chamaecyparissus L. & poor \\
\hline 151 & Erica Alexandrina Lob. seu sanamunda vulgo muffulena & Thymelaea hirsuta (L.) Endl. & good \\
\hline 152 & / & / & bad \\
\hline 152 & $\begin{array}{l}\text { Brassica sylvestris perfoliata Dod. Brassica campestris } \\
\text { perfoliata flore aureo Casp. Beguini }\end{array}$ & Brassica rapa subsp. campestris (L.) A. R. Clapham & poor \\
\hline 152 & ?vulgo ruvetto di san francisco & Rubus sp. & good \\
\hline 153 & Caucalis M. & Anthriscus sp. & good \\
\hline 153 & $\begin{array}{l}\text { Caltha vulgaris Casp. B. calendula Dod. Ital. Fiore di ogni mese } \\
\text { horologio de i contadini }\end{array}$ & Calendula stellata Cav. & good \\
\hline 153 & Solanus lethale Belladonna Clusij & Lycium chinense Mill. & poor \\
\hline 154 & Matricaria? & / & good \\
\hline 154 & Serratula sic. Gentil Galla & Salvia sp. & good \\
\hline 154 & $\begin{array}{l}\text { Smyrnius Matth. vulgo lisciandri olosatrus bad Ital. Smirnio } \\
\text { macedone Spag. Apio macedonico }\end{array}$ & Smyrnium olusatrum L. & poor \\
\hline 155 & Pinus sativa vulgo Pignu & Pinus halepensis Mill. & good \\
\hline 155 & Hypofillus & Ruscus hypophyllum L. & good \\
\hline 155 & Sphacelus verus Theophr. Salvia minore Mat. & Salvia fruticosa Mill. & good \\
\hline 156 & Palma arbor? & Phoenix dactylifera L. & good \\
\hline 156 & Hemeris Etymodris vulgo agliandra & Quercus robur L. & good \\
\hline 157 & Ilex major Matt. & Ilex aquifolium L. & good \\
\hline 157 & Lentischus Mat. vulgo Stinco? & Pistacia lentiscus L. & poor \\
\hline 157 & Polium montanum & Teucrium capitatum L. & good \\
\hline 158 & ? Papyrus gladiolus India? & Canna indica L. & poor \\
\hline 158 & Ranunculus tertius? & Ranunculus bullatus L. & bad \\
\hline 158 & Ranunculus lusitanicus Dodonei Ranunculus humili & Ranunculus bullatus L. & bad \\
\hline 158 & / & Ranunculus bullatus L. & bad \\
\hline 158 & Ranunculus? & Ranunculus bullatus $\mathrm{L}$. & bad \\
\hline 159 & $\begin{array}{l}\text { Clematis } 3^{\circ} \text { Boetica Matth. sive vitis silvestris Dodonei flammola } \\
\text { perperas? }\end{array}$ & Clematis cirrhosa L. & poor \\
\hline 159 & Melissophyllum Melissa Dios. Mat.Citronella? & Melissa officinalis L. & poor \\
\hline 159 & / & Saponaria officinalis L. & good \\
\hline 160 & / & / & poor \\
\hline 160 & Arthemisia? & Artemisia verlotiorum Lamotte & good \\
\hline 160 & $\begin{array}{l}\text { Nerium sive Rhododaphnem Plin. Rosas laureas Apulei Ital. Rosa } \\
\text { lauro et oleandro vulgo landru }\end{array}$ & Nerium oleander L. & good \\
\hline 161 & Papyrus ex Sicilia ? ciperialis Cyperi Bocc. Descrip. & Cyperus papyrus L. & good \\
\hline 161 & Narcissus verus luteus G.B. & Narcissus tazetta L. & good \\
\hline 161 & Polium montanum & Teucrium polium L. & good \\
\hline
\end{tabular}




\begin{tabular}{clll}
$\begin{array}{c}\text { Herbarium } \\
\text { sheet number }\end{array}$ & \multicolumn{1}{c}{$\begin{array}{c}\text { Plant name as indicated } \\
\text { in the Herbarium }\end{array}$} & \multicolumn{1}{c}{$\begin{array}{c}\text { Current taxonomic identification } \\
\text { preservation }\end{array}$} \\
\hline 162 & Amarantus purpureus sativo ? Lob. Blitus maximus Ch. & Amaranthus retroflexus L. & good \\
162 & Amarantus ? folias simplici spicata panniculata Lobel & Amaranthus sp. & good \\
162 & Papaver cornutus Sic. Cauliceddi marini & Glaucium corniculatum (L.) Rudolph & poor \\
162 & Panica ? sive panico ? & Panicum sp. & good \\
163 & Plumbago Herba Sant'Antonis vulgo ? cattiva & $/$ & good \\
163 & Hedera Mat. & Hedera helix L. & poor \\
\hline 163 & / & Heliotropium supinum L. & \\
\hline
\end{tabular}




\section{Appendix 1. - Taxonomic index.}

Abies alba Mill.

Acacia sp.

Acanthus mollis L. subsp. mollis

Acer campestre L.

Achillea maritima (L.) Ehrend. \& Y. P. Guo

Achillea millefolium $\mathrm{L}$.

Achillea sp.

Acinos alpinus (L.) Moench

Aconitum sp.

Adenostyles sp.

Adiantum capillus-veneris L.

Adonis annua L. subsp. annua

Agrostemma githago L.

Ajuga chamaepitys (L.) Schreb.

Ajuga orientalis L.

Alchemilla sp.

Allium chamaemoly L.

Allium siculum Ucria

Allium subvillosum Salzm. ex Schult. \& Schult. f.

Althaea officinalis $\mathrm{L}$.

Althea hirsuta $\mathrm{L}$.

Alyssum sp.

Amaranthus deflexus L.

Amaranthus retroflexus $L$.

Amaranthus sp.

Ammi sp.

Anagyris foetida L.

Anchusa sp.

Anemone apennina $\mathrm{L}$.

Angelica sylvestris $\mathrm{L}$.

Anisantha madritensis (L.) Nevski

Antennaria dioica (L.) Gaertn.

Anthemis cotula L.

Anthemis sp.

Anthriscus cerefolium (L.) Hoffm.

Anthriscus sp.

Anthyllis barba-jovis L.

Anthyllis vulneraria $\mathrm{L}$.

Antirrhinum majus L.

Apium sp.

Aquilegia vulgaris $\mathrm{L}$.

Arabis alpina subsp. caucasica (Willd.) Briq.

Arabis turrita $\mathrm{L}$.

Arbutus unedo L.

Arctium minus (Hill) Bernh.

Aremonia agrimonioides (L.) DC.

Arisarum vulgare $\mathrm{O}$. Targ. Tozz.

Aristolochia sicula Tineo

Artemisia absinthium L.

Artemisia pontica L.

Artemisia verlotiorum Lamotte

Arundo donax L.

Asparagus albus L.

Asparagus officinalis L.

Asperula aristata L. f.
Asperula arvensis L.

Asplenium adiantum-nigrum $\mathrm{L}$.

Asplenium ceterach L.

Asplenium ruta-muraria $\mathrm{L}$.

Asplenium scolopendrium L.

Asplenium trichomanes $\mathrm{L}$.

Aster cf. amellus L.

Astracantha sicula (Raf.) Greuter

Athamanta sp.

Atractylis cancellata $\mathrm{L}$.

Atriplex halimus L.

Atriplex prostrata DC.

Ballota pseudodictamnus (L.) Benth.

Bellardia trixago (L.) All.

Bellis perennis $\mathrm{L}$.

Biarum tenuifolium (L.) Schott

Biscutella lyrata $\mathrm{L}$.

Bituminaria bituminosa (L.) C. H. Stirt.

Blechnum spicant (L.) Roth

Borago officinalis $L$.

Brassica rapa subsp. campestris (L.) A. R. Clapham

Briza maxima L.

Briza minor L.

Bryonia cretica subsp. dioica (Jacq.) Tutin

Bubon macedonicum L.

Buglossoides arvensis (L.) I. M. Johnst.

Bupleurum fruticosum $\mathrm{L}$.

Cachrys sicula L.

Calendula stellata Cav.

Calystegia soldanella (L.) Roem. \& Schult.

Campanula dichotoma L.

Camphorosma monspeliaca $\mathrm{L}$.

Canna indica $\mathrm{L}$.

Carduus defloratus L.

Carduus pycnocephalus L.

Carex cf. vulpina L.

Carex cuprina (Heuff.) A. Kern.

Carex sp.

Carlina sicula Ten.

Carthamus pinnatus Desf.

Cassia sophera L.

Celtis australis L.

Centaurea calcitrapa L.

Centaurea seridis subsp. sonchifolia (L.) Greuter

Centaurea sp.

Centaurium erythraea Rafn

Cerastium arvense $\mathrm{L}$.

Cerastium sp.

Cerastium tomentosum L.

Cercis siliquastrum $\mathrm{L}$.

Cerinthe major $\mathrm{L}$.

Chamaemelum fuscatum (Brot.) Vasc.

Chelidonium majus L.
Chenopodium murale L.

Chenopodium vulvaria $\mathrm{L}$.

Chrozophora tinctoria (L.) A. Juss.

Circaea lutetiana $\mathrm{L}$.

Cistus incanus L.

Cistus monspeliensis L.

Citrus limon (L.) Burm. f.

Clematis cirrhosa L.

Clematis integrifolia $\mathrm{L}$.

Clematis sp.

Clematis vitalba $\mathrm{L}$.

Clinopodium vulgare $\mathrm{L}$.

Coix lacryma-jobi L.

Conium maculatum L.

Consolida sp.

Convallaria majalis L.

Convolvulus cneorum L.

Crambe hispanica L.

Crithmum maritimum L.

Crucianella maritima $\mathrm{L}$.

Cuscuta epithymum (L.) L.

Cyanus segetum Hill

Cynanchum acutum L.

Cynosurus echinatus L.

Cyperus capitatus Vand.

Cyperus papyrus L.

Cyperus sp.

Cytisus villosus Pourr.

Daphne alpina $\mathrm{L}$.

Delphinium fissum Waldst. \& Kit.

Delphinium sp.

Delphinium staphysagria L.

Dianthus deltoides L.

Dictamnus albus L.

Diplotaxis erucoides (L.) DC.

Dorycnium hirsutum (L.) Ser.

Dorycnium pentaphyllum Scop.

Dryopteris filix-mas (L.) Schott

Ecballium elaterium (L.) A. Rich.

Elaeagnus angustifolia $\mathrm{L}$.

Emex spinosa (L.) Campd.

Epilobium lanceolatum Sebast. \& Mauri

Epimedium alpinum L.

Eragrostis cilianensis (All.) Janch.

Erica multiflora L.

Erigeron sp.

Erodium moschatum (L.) L'Hér.

Erysimum cheiri (L.) Crantz

Euphorbia chamaesyce L.

Euphorbia paralias L.

Euphorbia sp.

Falcaria vulgaris Bernh.

Falcata legousia (Ten.) Janch.

Fedia cornucopiae (L.) Gaertn. 
Filipendula vulgaris Moench

Fragaria vesca L.

Fraxinus ornus L.

Fumana thymifolia (L.) Spach ex Webb.

Fumaria sp.

Galega officinalis L.

Galium aparine L.

Galium rotundifolium L.

Genista gasparrini (Guss.) C. Presl

Genista monspessulana (L.) L. A. S. Johnson

Gentiana clusii E. P. Perrier \& Songeon

Geranium pyrenaicum Burm. f.

Geranium robertianum L.

Geranium sp.

Geranium versicolor L.

Geum montanum L.

Geum urbanum L.

Gladiolus sp.

Glaucium corniculatum (L.) Rudolph

Glaucium flavum Crantz

Glebionis segetum (L.) Fourr.

Gomphocarpus fruticosus (L.) W. T. Aiton

Hedera helix L.

Hedysarum coronarium L.

Hedysarum canadense L.

Helianthus tuberosus L.

Helichrysum pendulum (C. Presl) C. PresI

Helichrysum sp.

Heliotropium europaeum L.

Heliotropium supinum L.

Helleborus sp.

Herniaria glabra subsp. nebrodensis Nyman

Hibiscus rosa-sinensis L.

Hieracium sp.

Hippocrepis emerus (L.) Lassen subsp. emerus

Horminum pyrenaicum L.

Humulus lupulus L.

Hypericum perfoliatum $L$.

Iberis semperflorens $L$.

Ilex aquifolium L.

Impatiens sp.

Inula conyzae (Griess.) DC.

Inula helenium L.

Inula oculus-christi L.

Iris planifolia (Mill.) Fiori \& Paol.

Jacobaea ambigua (Biv.) Pelser \& Veldkamp

Juniperus cf. sabina L.

Juniperus phoenicea $\mathrm{L}$.

Lamium galeobdolon (L.) Crantz

Lamium garganicum $\mathrm{L}$.

Lamium sp.

Lathyrus aphaca L.

Lathyrus gorgonii Parl.

Lathyrus ochrus (L.) DC.

Lathyrus sp.

Laurus sp.
Lavandula angustifolia Mill.

Lavandula dentata $\mathrm{L}$.

Lavandula sp.

Lavandula stoechas L.

Legousia speculum-veneris (L.) Chaix

Leontodon tuberosum $\mathrm{L}$.

Leonurus cardiaca L.

Ligustrum vulgare $\mathrm{L}$.

Limonium narbonense Mill.

Limonium sp.

Linaria purpurea (L.) Mill.

Linaria reflexa (L.) Desf.

Linum usitatissimum $\mathrm{L}$.

Lithospermum sp.

Lomelosia cretica (L.) Greuter \& Burdet

Lonas annua (L.) Vines \& Druce

Lonicera etrusca Santi

Lotus corniculatus L.

Lotus cytisoides L.

Lotus tetragonolobus L.

Lycium chinense Mill.

Lycopus europaeus $L$.

Lysimachia nummularia $\mathrm{L}$.

Lythrum junceum Banks \& Sol.

Malva arborea (L.) Webb \& Berthel.

Malva cretica Cav.

Matthiola tricuspidata (L.) R. Br.

Medicago intertexta (L.) Mill.

Medicago marina $\mathrm{L}$.

Medicago orbicularis (L.) Bartal.

Medicago polymorpha $\mathrm{L}$.

Medicago turbinata (L.) All.

Melampyrum arvense $\mathrm{L}$.

Melampyrum sp.

Melilotus infestus Guss.

Melilotus italicus (L.) Lam.

Melissa officinalis L.

Melissa sp.

Melittis melissophyllum subsp. albida (Guss.) P. W. Ball

Mentha spicata L.

Mercurialis annua $\mathrm{L}$.

Meum athamanticum Jacq.

Micromeria graeca (L.) Benth.

Micromeria juliana (L.) Benth. ex Rchb.

Micromeria sp.

Minuartia verna (L.) Hiern

Moricandia arvensis (L.) DC.

Myosotis sp.

Myrtus communis L.

Narcissus poeticus L.

Narcissus tazetta L.

Nepeta cataria L.

Nerium oleander L.

Nicotiana tabacum L.

Nigella damascena $\mathrm{L}$.

Ochlopoa annua (L.) H. Scholz
Odontites luteus (L.) Clairv.

Oenanthe pimpinelloides L.

Omphalodes linifolia (L.) Moench

Ononis natrix L. subsp. natrix

Ononis natrix subsp. ramosissima (Desf.) Batt.

Ononis oligophylla Ten.

Ononis sp.

Orchis sp.

Origanum dictamnus $\mathrm{L}$.

Origanum onites $\mathrm{L}$.

Origanum vulgare L.

Orobanche rapum-genistae Thuill.

Osmunda regalis $\mathrm{L}$.

Paeonia mascula (L.) Mill.

Paliurus spina-christi Mill.

Panicum sp.

Parentucellia latifolia (L.) Caruel

Paronychia argentea Lam.

Paronychia echinatula Chater

Pastinaca sativa L.

Pelargonium sp.

Persicaria maculosa (L.) Gray

Petasites pyrenaicus (L.) G. López

Peucedanum sp.

Phagnalon saxatile (L.) Cass.

Phagnalon sordidum (L.) Rchb.

Phlomis fruticosa $\mathrm{L}$.

Phlomis herba-venti $\mathrm{L}$.

Phoenix dactylifera $\mathrm{L}$.

Phragmites australis (Cav.) Steud.

Phyla nodiflora (L.) Greene

Physalis alkekengi L.

Phytolacca americana L.

Picnomon acarna (L.) Cass.

Pinus halepensis Mill.

Pistacia lentiscus L.

Pistacia terebinthus $\mathrm{L}$.

Pistacia vera $\mathrm{L}$.

Pisum sativum L.

Plantago afra $\mathrm{L}$.

Plantago coronopus L.

Plantago lagopus L.

Plantago major L.

Plantago sempervirens Crantz

Poa sp.

Polycarpon tetraphyllum (L.) L.

Polygala preslii Spreng.

Polygonum maritimum $\mathrm{L}$.

Polygonum sp.

Potentilla sp.

Primula acaulis (L.) L.

Prunus mahaleb L.

Pulmonaria sp.

Quercus robur L.

Ranunculus bullatus $\mathrm{L}$.

Ranunculus flammula $\mathrm{L}$. 
Ranunculus sp.

Reichardia picroides (L.) Roth

Reseda alba L.

Rhamnus catharticus L.

Ribes sp.

Ricinus communis L.

Rosa moschata Herrm.

Rubia peregrina L.

Rubia tinctorum L.

Rubus canescens DC.

Rubus sp.

Rumex bucephalophorus L.

Rumex lunaria L.

Rumex obtusifolius L.

Rumex sp.

Ruscus hypophyllum L.

Ruta chalepensis L.

Salicornia sp.

Salix sp.

Salsola oppositifolia Desf.

Salvia aethiopis $\mathrm{L}$.

Salvia fruticosa Mill.

Salvia officinalis $\mathrm{L}$.

Salvia pratensis L. subsp. pratensis

Salvia sclarea $\mathrm{L}$.

Salvia sp.

Salvia verbenaca $L$.

Sambucus ebulus L.

Sanguisorba minor Scop.

Sanicula europaea L.

Santolina chamaecyparissus L.

Saponaria officinalis $\mathrm{L}$.

Sarcopoterium spinosum (L.) Spach

Satureja sp.

Saxifraga tridactylites $\mathrm{L}$.

Scabiosa columbaria L.

Scandix pecten-veneris $L$.

Schinus molle L.

Scorzonera sp.

Scorzonera undulata subsp. deliciosa (Guss.)

$$
\text { Maire }
$$

Scrophularia nodosa L.

Scrophularia peregrina $\mathrm{L}$.

Scutellaria columnae All.

Senecio sp.

Senecio vulgaris $L$.

Seseli tortuosum L.

Sideritis sp.

Silene dioica (L.) Clairv.

Silene sp.

Sisymbrium irio L.

Smilax aspera $\mathrm{L}$.

Smyrnium olusatrum L.

Smyrnium perfoliatum subsp. rotundifolium (Mill.) Bonnier \& Layens

Solanum dulcamara $\mathrm{L}$.
Solanum linnaeanum Hepper \& P.-M. L. Jaeger

Solanum sp.

Solidago virga-aurea $\mathrm{L}$.

Sonchus bulbosus (L.) N. Kilian \& Greuter Sonchus oleraceus L.

Sorbus aria (L.) Crantz

Sorbus aucuparia L.

Spergularia marina (L.) Griseb.

Spergularia rubra (L.) J. Presl \& C. Presl

Sporobolus pungens (Schreb.) Kunth

Stachys ocymastrum (L.) Briq.

Stachys officinalis (L.) Trevis.

Stachys sp.

Styrax officinalis L.

Succisa pratensis Moench

Symphytum bulbosum K. F. Schimp.

Symphytum tuberosum L.

Tagetes erecta $\mathrm{L}$.

Tanacetum balsamita $\mathrm{L}$.

Tanacetum corymbosum (L.) Sch. Bip.

Tanacetum sp.

Tanacetum vulgare $\mathrm{L}$.

Taxus baccata L.

Teucrium capitatum L.

Teucrium chamaedrys L.

Teucrium flavum $\mathrm{L}$.

Teucrium fruticans $L$.

Teucrium polium L.

Thalictrum aquilegiifolium L.

Thalictrum minus $\mathrm{L}$.

Thalictrum sp.

Thymbra capitata (L.) Cav.

Thymelaea hirsuta (L.) Endl.

Thymelaea passerina (L.) Coss. \& Germ.

Thymus serpyllum $\mathrm{L}$.

Thymus striatus Vahl

Tragopogon crocifolius L.

Trifolium arvense L.

Trifolium badium Schreb.

Tropaeolum majus L.

Tussilago farfara $\mathrm{L}$.

Urtica dioica L.

Urtica sp.

Valerianella sp.

Verbascum blattaria L.

Verbascum sinuatum L.

Verbascum thapsus $\mathrm{L}$.

Verbena officinalis L.

Veronica anagallis-aquatica $\mathrm{L}$.

Veronica arvensis L.

Veronica sp.

Vicia melanops Sibth. \& Sm.

Vinca major L.

Vincetoxicum hirundinaria Medik.

Viola arvensis Murray

Viola cf. odorata L.
Viscum album L.

Vitex agnus-castus $\mathrm{L}$.

Vitis laciniosa L.

Withania somnifera (L.) Dunal

Xanthium strumarium L.

Xeranthemum inapertum (L.) Mill.

Zea mays L.

Ziziphus lotus (L.) Lam. 